\title{
Elliptic genera and real Jacobi forms
}

\section{Sujay K. Ashok ${ }^{a}$ and Jan Troost ${ }^{b}$}

\author{
${ }^{a}$ Institute of Mathematical Sciences, \\ C.I.T Campus, Taramani, Chennai, India 600113 \\ ${ }^{b}$ Laboratoire de Physique Théorique, ${ }^{1}$ Ecole Normale Supérieure, \\ 24 rue Lhomond, F-75231 Paris Cedex 05, France \\ E-mail: sashok@imsc.res.in, troost@lpt.ens.fr
}

ABSTRACT: We construct real Jacobi forms with matrix index using path integrals. The path integral expressions represent elliptic genera of two-dimensional $\mathcal{N}=(2,2)$ supersymmetric theories. They arise in a family labeled by two integers $N$ and $k$ which determine the central charge of the infrared fixed point through the formula $c=3 N(1+2 N / k)$. We decompose the real Jacobi form into a mock modular form and a term arising from the continuous spectrum of the conformal field theory. For a given $N$ and $k$ we argue that the Jacobi form represents the elliptic genus of a theory defined on a $2 N$ dimensional linear dilaton background with $\mathrm{U}(N)$ isometry, an asymptotic circle of radius $\sqrt{k \alpha^{\prime}}$ and linear dilaton slope $N \sqrt{2 / k}$. We also present formulas for the elliptic genera of their orbifolds.

Keywords: Conformal Field Models in String Theory, Extended Supersymmetry, Conformal and W Symmetry

ARXIV EPRINT: 1310.2124

\footnotetext{
${ }^{1}$ Unité Mixte du CNRS et de l'Ecole Normale Supérieure associée à l'université Pierre et Marie Curie 6, UMR 8549.
} 


\section{Contents}

1 Introduction 2

2 A path integral elliptic genus 3

2.1 The cigar elliptic genus 3

2.2 A proposed path integral generalization 3

2.3 A real Jacobi form of matrix index 4

3 The mock modular form $\quad 6$

3.1 The Hamiltonian viewpoint 6

3.2 The holomorphic, mock modular form 8

$\begin{array}{lll}3.3 & \text { Further analysis of the holomorphic part } & 10\end{array}$

$\begin{array}{lll}3.3 .1 & \text { Character decomposition } & 10\end{array}$

$\begin{array}{ll}\text { 3.3.2 A contour integral representation } & 12\end{array}$

3.3.3 The Witten index 13

$\begin{array}{ll}3.4 & \text { The modular completion of the mock modular form } \\ \end{array}$

$\begin{array}{lll}3.4 .1 & \text { A succinct expression } & 13\end{array}$

$\begin{array}{ll}\text { 3.4.2 Observations on the modular completion } & 14\end{array}$

4 Asymptotically linear dilaton conformal field theory $\quad 16$

$\begin{array}{lll}4.1 & \text { Asymptotically linear dilaton target spaces } & 16\end{array}$

$\begin{array}{ll}\text { 4.2 The gauged linear sigma model path integral } & 17\end{array}$

$\begin{array}{ll}4.3 \text { The count of wound bound states } & 18\end{array}$

4.3.1 Counting bound states from supersymmetric quantum mechanics 18

$\begin{array}{ll}\text { 4.3.2 Matching wound bound states } & 20\end{array}$

4.4 Features of the asymptotic geometry and susy algebra 22

$5 \quad$ Elliptic genus of generalized Liouville theories $\quad 23$

5.1 The mock modular form and its completion 23

5.1.1 The completion 24

5.1.2 Integral representation 24

5.1.3 Poincaré polynomial 24

5.1.4 Character interpretation 25

$\begin{array}{lll}5.2 \text { Orbifolds } 25 & 25\end{array}$

6 Conclusions 26

$\begin{array}{lr}\text { A Formulas } & \mathbf{2 8}\end{array}$

$\begin{array}{lll}\text { A.1 Definitions and properties } & 28\end{array}$

$\begin{array}{lll}\text { A.2 Identities and their proof } & 28\end{array}$ 


\section{Introduction}

Despite a long and rich history, quantum field theory continues to develop in surprising ways. In particular, we continue to discover new classes of conformal field theories in various dimensions with novel properties. While we do not always have a handle on the full spectrum of the conformal field theory, or even a Lagrangian description, we can often give a catalog of detailed properties of the conformal field theory through other means.

In this paper, we concentrate on conformal field theories in two dimensions with $\mathcal{N}=$ $(2,2)$ supersymmetry. For these theories, we have characterizing properties like the central charge, the Witten index, the spectrum of chiral primaries, the elliptic genus, three-point functions, boundaries preserving conformal symmetry etc. Often we are only able to specify part of the characterizing properties of the conformal field theory. In this paper we propose path integral expressions for the elliptic genus of certain conformal field theories, and we give good evidence for an identification of the conformal field theories in question.

The elliptic genus is a weighted trace that captures short representations in the spectrum [1-5], as well as rough characteristics of the continuum [6]. In the Hamiltonian formalism, it is given by a trace over the Hilbert space

$$
\chi(\tau, \alpha)=\operatorname{Tr}(-1)^{F} z^{J_{0}} q^{L_{0}-\frac{c}{24}} \bar{q}^{\bar{L}_{0}-\frac{c}{24}} .
$$

Here, $q=e^{2 \pi i \tau}$ and $z=e^{2 \pi i \alpha}$. The scaling operator $L_{0}$ measures the conformal dimension of left movers and the operator $J_{0}$ measures the left moving $R$-charge. In cases for which the spectrum is discrete, the insertion $(-1)^{F}$ projects onto the right moving ground states and the resulting elliptic genera are holomorphic Jacobi forms, i.e. they have well defined modular and elliptic properties and are holomorphic functions of $q$ and $z$. However, when the conformal field theory in question has a continuum of states the elliptic genus is typically not holomorphic [6]. It is the modular completion of a mock Jacobi form $[7,8]$. The mock Jacobi form is holomorphic and arises from right-moving ground state contributions. The modular completion arises from the continuum, which contributes even though the rightmovers are not in the ground state. The resulting expression is a real Jacobi form.

The analysis of $[6]$ was generalized in $[9,10]$ in which a twisted elliptic genus of the supersymmetric coset $\mathrm{SL}(2, \mathbb{R}) / \mathrm{U}(1)$ or cigar conformal field theory was calculated from the path integral formalism. It was made clear in $[6,10,11]$ that the non-holomorphic contribution arises due to a mismatch in the spectral density of bosons and fermions in the continuum sector. The mismatch can be characterized in terms of the asymptotic expression for the supercharge.

In this article we propose path integral expressions for twisted elliptic genera that are generalizations of the path integral expression for the cigar and Liouville conformal field theory $[6,10]$. The central charge of the conformal field theories is $c=3 N(1+2 N / k)$. They are further characterized by $\mathcal{N}=2$ superconformal symmetry and at least a $\mathrm{U}(1)^{N}$ global symmetry. In mathematical terms, our expression for the elliptic genus is described as the modular completion of a mock Jacobi form with matrix index. The Jacobi forms depend on a chemical potential for $R$-charge and $N$ chemical potentials for the global symmetry group $\mathrm{U}(1)^{N}$. 
Finally, we argue for the identification of the conformal field theory that gives rise to these elliptic genera. The generalization of the cigar elliptic genus is the elliptic genus of linear dilaton spaces of dimension $2 N$, discovered in [12] and further analyzed in $[13,14]$ as well as [11]. In particular, we are able to provide a detailed identification of contributions to the elliptic genus by the wound, bound strings of [11].

This paper is organized as follows. In section 2, we briefly review relevant properties of the elliptic genus of the cigar conformal field theory. We propose our generalization to a class of models labeled by two integers $k$ and $N$. The proposal has good modular and elliptic properties, namely it is a real Jacobi form with matrix index. In section 3 we derive the holomorphic component of the elliptic genus, and provide various forms of it and a detailed decomposition in terms of characters. We also identify the covariantization provided by the path integral expression. In section 4, we compare the properties of the proposed elliptic genus with properties of asymptotic linear dilaton models and heuristically connect to a gauged linear sigma model description of these models. In section 5 , we propose path integral expressions for generalizations of $\mathcal{N}=2$ Liouville theories and show that a diagonal orbifold leads back to the generalized cigar elliptic genus. We conclude in section 6 .

\section{$2 \quad$ A path integral elliptic genus}

In this section, we review the path integral result for the elliptic genus of the $\mathcal{N}=2$ superconformal cigar conformal field theory $[6,9,10]$. We then generalize the path integral expression to a proposal for the elliptic genus for a two-dimensional $\mathcal{N}=2$ superconformal field theory in two dimensions labeled by an extra integer $N$ and with at least a $\mathrm{U}(1)^{N}$ global symmetry. We show that our proposal is a real Jacobi form of matrix index. It is a function of a modular parameter $q=e^{2 \pi i \tau}$, an $R$-charge fugacity $z=e^{2 \pi i \alpha}$ as well as $N$ chemical potentials $y_{i}=e^{2 \pi i \beta_{i}}$.

\subsection{The cigar elliptic genus}

The path integral calculation of the cigar elliptic genus gives rise to the result:

$$
\chi_{\cos }(\tau, \alpha)=k \int_{0}^{1} d s_{1} d s_{2} \sum_{m, w \in \mathbb{Z}}\left[\frac{\theta_{11}\left(\tau, s_{1} \tau+s_{2}-\alpha-\frac{\alpha}{k}\right)}{\theta_{11}\left(\tau, s_{1} \tau+s_{2}-\frac{\alpha}{k}\right)}\right] e^{2 \pi i \alpha w} e^{-\frac{\pi k}{\tau_{2}}\left|m+w \tau+\left(s_{1} \tau+s_{2}\right)\right|^{2}} .
$$

The calculation of the path integral in the end boils down to the contribution of elementary ingredients [10]. There is the contribution of the zero modes of a charged complex boson of radius $\sqrt{k \alpha^{\prime}}$, a charged complex fermion of charge one, a complex boson of charge one, and Wilson lines $s_{1}$ and $s_{2}$ for the corresponding U(1) gauge group. The complex boson has $R$-charge $1+1 / k$ while the complex fermion has $R$-charge $1 / k$. The phase $e^{2 \pi i \alpha w}$ ensures periodicity in the Wilson line $s_{1}$ and can be argued on the basis of modular covariance or a chiral fermion anomaly. These ingredients lead to the final result (2.1).

\subsection{A proposed path integral generalization}

We propose the following generalized path integral result:

$$
\chi_{N}\left(\tau, \alpha, \beta_{i}\right)=k \int_{0}^{1} d s_{1} d s_{2} \sum_{m, w \in \mathbb{Z}} \prod_{i=1}^{N}\left[\frac{\theta_{11}\left(\tau, s_{1} \tau+s_{2}-\alpha-\frac{N \alpha}{k}+\beta_{i}\right)}{\theta_{11}\left(\tau, s_{1} \tau+s_{2}-\frac{N \alpha}{k}+\beta_{i}\right)}\right] e^{2 \pi i N \alpha w} e^{-\frac{\pi k}{\tau_{2}}\left|m+w \tau+\left(s_{1} \tau+s_{2}\right)\right|^{2}},
$$


where the case $N=1$ correspond to the cigar elliptic genus (2.1). We can identify the final ingredients that make up this path integral. We have a model with $\mathrm{U}(1)$ gauge field under which we have $N$ charged complex fermions and bosons, each carrying charge 1 . We also have the zero mode of a charged boson of radius $\sqrt{k \alpha^{\prime}}$. The complex bosons have $R$-charge $1+N / k$, and the $R$-charge of the fermions differs from these by one. The anomalous phase factor again ensures periodicity in the holonomy $s_{1}$ of the U(1) gauge field. Each complex boson and fermion is charged under a global $\mathrm{U}(1)^{N}$ symmetry.

Another useful perspective on the path integral arises when we rewrite the expression in terms of holonomies taking values on the real line:

$$
\chi_{N}\left(\tau, \alpha, \beta_{i}\right)=k \int_{-\infty}^{+\infty} d s_{1} d s_{2} \prod_{i=1}^{N}\left[\frac{\theta_{11}\left(\tau, s_{1} \tau+s_{2}-\alpha-\frac{N \alpha}{k}+\beta_{i}\right)}{\theta_{11}\left(\tau, s_{1} \tau+s_{2}-\frac{N \alpha}{k}+\beta_{i}\right)}\right] e^{-\frac{\pi k}{\tau_{2}}\left|s_{1} \tau+s_{2}\right|^{2}} .
$$

We note that near a multiple pole of order $m$ of the ratio of $\theta_{11}$ functions, we can write an approximation to the integral in terms of a coordinate $z$ near the multiple pole as:

$$
\int d z d \bar{z} \frac{1}{z^{m}} \sum_{n, \bar{n} \geq 0} c_{n, \bar{n}} z^{n} \bar{z}^{\bar{n}}
$$

The angular integration will give zero unless $n-m=\bar{n} \geq 0$, and therefore our integral expression is free of divergences.

In summary, we propose that this expression is the result of computing a generalized elliptic genus of a two-dimensional conformal field theory with $\mathcal{N}=2$ superconformal symmetry and at least a $\mathrm{U}(1)^{N}$ global symmetry through path integral means. We give evidence for what this theory is in section 4. In this section we take equation (2.2) as our starting point and analyze its modular and elliptic properties.

\subsection{A real Jacobi form of matrix index}

After double Poisson resummation, we obtain an expression with which it is easier to check the modular properties:

$$
\begin{aligned}
& \chi_{N}\left(\tau, \alpha, \beta_{i}\right)=\int_{0}^{1} d s_{1} d s_{2} \sum_{m, w \in \mathbb{Z}} \prod_{i=1}^{N}\left[\frac{\theta_{11}\left(\tau, s_{1} \tau+s_{2}-\alpha-\frac{N \alpha}{k}+\beta_{i}\right)}{\theta_{11}\left(\tau, s_{1} \tau+s_{2}-\frac{N \alpha}{k}+\beta_{i}\right)}\right] \\
& \times e^{-2 \pi i s_{2} w} e^{2 \pi i s_{1}(m-N \alpha)} e^{-\frac{\pi}{k \tau_{2}}|m-N \alpha+w \tau|^{2}} .
\end{aligned}
$$

We consider the behavior of the elliptic genus under the action of the modular group on $\tau$. Since this operation interchanges the cycles of the torus, it must be accompanied by the corresponding transformation of the holonomies and the winding numbers. One can check that the following operation leaves the expression invariant:

$$
\tau \rightarrow \tau+1 \quad m \rightarrow m-w \quad s_{2} \rightarrow s_{2}-s_{1} .
$$

The measure factor does not change and we used that the integrand is periodic in the holonomies. This shows invariance under the $T$ transformation. In order to obtain the 
transformation of the elliptic genus under the $S$-transformation

$$
\begin{aligned}
& \tau \rightarrow-\frac{1}{\tau} \quad \alpha \rightarrow \frac{\alpha}{\tau} \quad \beta_{i} \rightarrow \frac{\beta_{i}}{\tau} \\
& s_{1} \rightarrow-s_{2} \quad s_{2} \rightarrow s_{1} \quad w \rightarrow-m \quad m \rightarrow w,
\end{aligned}
$$

we use the transformation of the Jacobi theta function

$$
\theta_{11}\left(-\frac{1}{\tau}, \frac{\alpha}{\tau}\right)=-i(-i \tau)^{\frac{1}{2}} e^{\frac{\pi i z^{2}}{\tau}} \theta_{11}(\tau, \alpha)
$$

The phase factor that one picks up is given by:

$$
\begin{aligned}
& \frac{\pi i}{\tau} \sum_{i=1}^{N}\left(-2 \alpha\left(s_{1} \tau+s_{2}-\frac{N \alpha}{k}+\beta_{i}\right)+\alpha^{2}\right)+\frac{2 \pi i N \alpha}{\tau}\left(s_{1} \tau+s_{2}\right) \\
& =\frac{\pi i \alpha^{2}}{\tau} \frac{c}{3}-\frac{\pi i}{\tau} 2 \alpha \sum_{i=1}^{N} \beta_{i}
\end{aligned}
$$

where we introduced the central charge parameter:

$$
c=3 N\left(1+\frac{2 N}{k}\right) .
$$

At this stage, we will suppose that the quantity:

$$
M=\frac{k}{N},
$$

is an integer, although this is not strictly necessary. If it is, our expression is elliptic in $\alpha$ where we can perform shifts by multiples of $M .{ }^{1}$ The parameters $\beta_{i}$ are elliptic in integer multiples of the periods of the torus. We summarize the modular and elliptic properties:

$$
\begin{aligned}
\chi_{N}\left(\tau+1, \alpha, \beta_{i}\right) & =\chi_{N}\left(\tau, \alpha, \beta_{i}\right) \\
\chi_{N}\left(-\frac{1}{\tau}, \frac{\alpha}{\tau}, \frac{\beta_{i}}{\tau}\right) & =e^{\frac{\pi i \frac{c}{3} \alpha^{2}}{\tau}} e^{-\frac{\pi i}{\tau} 2 \alpha \sum_{i} \beta_{i}} \chi_{N}\left(\tau, \alpha, \beta_{i}\right) \quad \text { where all } \beta_{i} \text { are rescaled. } \\
\chi_{N}\left(\tau, \alpha+M, \beta_{i}\right) & =(-1)^{k} \chi_{N}\left(\tau, \alpha, \beta_{i}\right) \\
\chi_{N}\left(\tau, \alpha+M \tau, \beta_{i}\right) & =(-1)^{k} e^{-\pi i \frac{c}{3}\left(M^{2} \tau+2 M \alpha\right)} e^{2 \pi i M \sum_{i} \beta_{i}} \chi_{N}\left(\tau, \alpha, \beta_{i}\right) \\
\chi_{N}\left(\tau, \alpha, \beta_{i}+1\right) & =\chi_{N}\left(\tau, \alpha, \beta_{i}\right) \\
\chi_{N}\left(\tau, \alpha, \beta_{i}+\tau\right) & =e^{2 \pi i \alpha} \chi_{N}\left(\tau, \alpha, \beta_{i}\right) \quad \text { where only a single } \beta_{i} \text { is shifted. }
\end{aligned}
$$

We have a real Jacobi form with matrix index (see e.g. $[15,16])$ given by

$$
\left(\begin{array}{cccc}
-\frac{c}{3} & 1 & 1 & \ldots \\
1 & 0 & 0 & \ldots \\
1 & 0 & 0 & \ldots \\
\ldots & \ldots & \ldots & \ldots
\end{array}\right)
$$

\footnotetext{
${ }^{1}$ If $M$ were not integer, we would continue the discussion in terms of shifts by multiples of $k$.
} 
We have made allowance for a parameter $\alpha$ that is normalized in accordance with standard physics conventions. If one wants to renormalize $\alpha$ to have periods $(1, \tau)$, one should rescale the entries in the table accordingly. Finally, we note the charge conjugation symmetry:

$$
\chi_{N}\left(\tau,-\alpha,-\beta_{i}\right)=\chi_{N}\left(\tau, \alpha, \beta_{i}\right) .
$$

In summary, we have a path integral expression for a real Jacobi form with matrix index. The Lagrangian perspective renders manifest the elliptic and modular properties of the elliptic genus.

\section{The mock modular form}

In this section, we would like to find a Hamiltonian interpretation of the path integral expression that we proposed in section 2. We wish to distinguish holomorphic contributions, arising from right-moving ground states in the underlying conformal field theory, and a remainder term that originates in the continuous part of the spectrum. We present an interpretation of the holomorphic contribution in terms of $\mathcal{N}=2$ superconformal algebra characters, and offer a rewriting in terms of a contour integral. The latter is reminiscent of the expressions for purely holomorphic elliptic genera arising from gauged linear sigma-models.

\subsection{The Hamiltonian viewpoint}

In the first few steps, we prepare the ground for an interpretation of our expression in terms of a physical state sum by going to Hamiltonian variables and executing the integral over the holonomies. We start by singly Poisson resumming our proposal to find after relabeling:

$$
\chi_{N}\left(\tau, \alpha, \beta_{i}\right)=\sqrt{k \tau_{2}} \int_{0}^{1} d s_{1,2} \sum_{n, w \in \mathbb{Z}} \prod_{i=1}^{N}\left[\frac{\theta_{11}\left(\tau, s_{1} \tau+s_{2}-\alpha-\frac{N \alpha}{k}+\beta_{i}\right)}{\theta_{11}\left(\tau, s_{1} \tau+s_{2}-\frac{N \alpha}{k}+\beta_{i}\right)}\right] e^{2 \pi i N \alpha w} e^{-2 \pi i s_{2} n} q^{\ell_{0}} \bar{q}^{\overline{\bar{c}}_{0}},
$$

where we introduced contributions $\ell_{0}$ and $\bar{\ell}_{0}$ to the conformal dimensions equal to

$$
\ell_{0}=\frac{\left(n-k\left(w+s_{1}\right)\right)^{2}}{4 k} \quad \bar{\ell}_{0}=\frac{\left(n+k\left(w+s_{1}\right)\right)^{2}}{4 k} .
$$

These correspond to momenta and winding of a boson of radius $\sqrt{k \alpha^{\prime}}$, twisted by the holonomies $s_{1,2}$ of the $\mathrm{U}(1)$ gauge field. We expand the denominator, using the formula

$$
\frac{1}{i \theta_{11}(\tau, \alpha)}=\frac{1}{\eta^{3}(\tau)} \sum_{r \in \mathbb{Z}} z^{r+\frac{1}{2}} S_{r}(q),
$$

where $q=e^{2 \pi i \tau}$ and $z=e^{2 \pi i \alpha}$ and

$$
S_{r}(q)=\sum_{n=0}^{\infty}(-1)^{n} q^{\frac{n(n+2 r+1)}{2}} .
$$

In the expression for the elliptic genus, we use this expansion for each theta function in the denominator; for each such term, the argument $z$ is given by

$$
x_{i}=q^{s_{1}} e^{2 \pi i s_{2}} z^{-\frac{N}{k}} y_{i}
$$


where $y_{i}=e^{2 \pi i \beta_{i}}$. As proven in appendix A.2, the expansion is valid when $|q|<\left|x_{i}\right|<1$ which we will assume from now on. Note in particular that when $\left|y_{i}\right|=|z|=1$, we have that $|q|<\left|q^{s_{1}}\right|<1$ for $s_{1}$ between 0 and 1 . Here, the decomposition of the holonomy plane into periodic variables $s_{1,2}$ and integer parts $(m, w)$ plays a crucial role. We also write the theta series in the numerator as:

$$
\theta_{11}(\tau, \alpha)=i \sum_{m \in \mathbb{Z}}(-1)^{m} q^{\frac{\left(m-\frac{1}{2}\right)^{2}}{2}} z^{-m+\frac{1}{2}}
$$

where, for each term in the product, the argument $z$ is given by

$$
z_{i}=q^{s_{1}} e^{2 \pi i s_{2}} z^{-1-\frac{N}{k}} y_{i}
$$

We then collect terms and perform the integral over the holonomy $s_{2}$ which imposes the constraint of gauge invariance on the physical state space:

$$
\sum_{i=1}^{N}\left(r_{i}-m_{i}+1\right)=n .
$$

The constraint leads to simplifications that give rise to:

$$
\begin{aligned}
\chi_{N}\left(\tau, \alpha, \beta_{i}\right)=(-1)^{N} \frac{\sqrt{k \tau_{2}}}{\eta^{3 N}(\tau)} \sum_{m_{i}, r_{i}, n, w} \int_{0}^{1} d s_{1}(-1)^{\sum_{i} m_{i}} q^{\frac{1}{2} \sum_{i}\left(m_{i}-\frac{1}{2}\right)^{2}} z^{\sum_{i}\left(m_{i}-\frac{1}{2}\right)} \\
q^{-n w} z^{N w-\frac{N n}{k}}(q \bar{q})^{\bar{\ell}_{0}} \delta_{\sum_{i}\left(r_{i}-m_{i}+1\right)-n} \prod_{i=1}^{N} y_{i}^{r_{i}-m_{i}+1} S_{r_{i}}(q) .
\end{aligned}
$$

We will henceforth omit writing the explicit argument of the function $S_{r}(q)$ in order to make the formulas less cumbersome. To linearize the integration over the second holonomy $s_{1}$, we introduce the integration over a variable $s$ that will have an interpretation as a non-compact (radial) momentum. We moreover introduce the right-moving momentum $v$ on the circle of radius $\sqrt{k \alpha^{\prime}}$ :

$$
v=n+k w
$$

We end up with

$$
\begin{gathered}
\chi_{N}\left(\tau, \alpha, \beta_{i}\right)=(-1)^{N} \frac{2 \tau_{2}}{\eta^{3 N}(\tau)} \sum_{m_{i}, r_{i}, v, w} \int_{-\infty}^{\infty} d s \int_{0}^{1} d s_{1}(-1)^{\sum_{i} m_{i}} q^{\frac{1}{2} \sum\left(m_{i}-\frac{1}{2}\right)^{2}} z^{\sum_{i}\left(m_{i}-\frac{1}{2}\right)} \\
q^{k w^{2}-v w} z^{N\left(2 w-\frac{v}{k}\right)}(q \bar{q})^{\frac{v^{2}}{4 k}+\frac{s^{2}}{k}+s_{1}\left(i s+\frac{v}{2}\right)} \delta_{\sum_{i}\left(r_{i}-m_{i}+1\right)-(v-k w)} \prod_{i=1}^{N} y_{i}^{r_{i}-m_{i}+1} S_{r_{i}} .
\end{gathered}
$$

Finally, performing the $s_{1}$ holonomy integral, we get:

$$
\begin{gathered}
\chi_{N}\left(\tau, \alpha, \beta_{i}\right)=(-1)^{N+1} \frac{1}{\pi \eta^{3 N}(\tau)} \sum_{m_{i}, r_{i}, v, w} \int_{-\infty}^{+\infty} \frac{d s}{2 i s+v}(-1)^{\sum_{i} m_{i}} q^{\frac{1}{2} \sum\left(m_{i}-\frac{1}{2}\right)^{2}} z^{\sum_{i}\left(m_{i}-\frac{1}{2}\right)} \\
q^{k w^{2}-v w} z^{N\left(2 w-\frac{v}{k}\right)}(q \bar{q})^{\frac{v^{2}}{4 k}+\frac{s^{2}}{k}}\left((q \bar{q})^{i s+\frac{v}{2}}-1\right) \delta_{\sum_{i}\left(r_{i}-m_{i}+1\right)-(v-k w)} \prod_{i=1}^{N} y_{i}^{r_{i}-m_{i}+1} S_{r_{i}} .
\end{gathered}
$$


We have prepared the ground for a state sum interpretation, by performing the integral over holonomies and introducing a non-compact radial momentum $s$. However, interpretation is still not straightforward since the expression exhibits an imaginary exponent of the modular parameter $q$, which we wish to avoid in a unitary state space sum. In what follows we perform a slight variation of the analysis in $[6,10]$ and extract both a holomorphic mock modular contribution and a remainder term from this expression, which exhibit exponents corresponding to real conformal dimensions.

\subsection{The holomorphic, mock modular form}

In equation (3.12) we can distinguish two terms. The first term has an imaginary exponent and is of the form:

$$
\begin{gathered}
\chi_{N, I}\left(\tau, \alpha, \beta_{i}\right)=(-1)^{N+1} \frac{1}{\pi \eta^{3 N}(\tau)} \sum_{m_{i}, r_{i}, v, w} \int_{\mathbb{R}+i \epsilon} \frac{d s}{2 i s+v}(-1)^{\sum_{i} m_{i}} q^{\frac{1}{2} \sum\left(m_{i}-\frac{1}{2}\right)^{2}} z^{\sum_{i}\left(m_{i}-\frac{1}{2}\right)} \\
q^{k w^{2}-v w} z^{N\left(2 w-\frac{v}{k}\right)}(q \bar{q})^{\frac{v^{2}}{4 k}+\frac{s^{2}}{k}}(q \bar{q})^{i s+\frac{v}{2}} \delta_{\sum_{i}\left(r_{i}-m_{i}+1\right)-(v-k w)} \prod_{i=1}^{N} y_{i}^{\left(r_{i}-m_{i}+1\right)} S_{r_{i}}, \quad
\end{gathered}
$$

while the second piece has real exponents and takes the form

$$
\begin{aligned}
\chi_{N, I I}\left(\tau, \alpha, \beta_{i}\right)= & (-1)^{N} \frac{1}{\pi \eta^{3 N}(\tau)} \sum_{m_{i}, r_{i}, v, w} \int_{\mathbb{R}+i \epsilon} \frac{d s}{2 i s+v}(-1)^{\sum_{i} m_{i}} q^{\frac{1}{2} \sum\left(m_{i}-\frac{1}{2}\right)^{2}} z^{\sum_{i}\left(m_{i}-\frac{1}{2}\right)} \\
& q^{k w^{2}-v w} z^{N\left(2 w-\frac{v}{k}\right)}(q \bar{q})^{\frac{v^{2}}{4 k}+\frac{s^{2}}{k}} \delta_{\sum_{i}\left(r_{i}-m_{i}+1\right)-(v-k w)} \prod_{i=1}^{N} y_{i}^{\left(r_{i}-m_{i}+1\right)} S_{r_{i}} .
\end{aligned}
$$

When separating the two terms, a regularization of the pole at $s=0=v$ is required. It introduces a minor ambiguity (related to the arbitrary separation between discretuum and continuum at zero radial momentum) of little consequence in the following. (See e.g. [6, 10].)

Our technique will be to shift the contour of the second piece (3.14) until part of it combines well with the first term, into a holomorphic discrete contribution. What is left of the second piece, we can then move back to the real contour of integration, guaranteeing that all contributions will have a real exponent.

We start with the second term and perform the following shifts in the integration and summation variables:

$$
v \rightarrow v+k \quad w \rightarrow w+1 \quad s \rightarrow s+\frac{i k}{2} .
$$

This part of the elliptic genus can then be written as

$$
\begin{gathered}
\chi_{N, I I}\left(\tau, \alpha, \beta_{i}\right)=\frac{(-1)^{N}}{\pi \eta^{3 N}(\tau)} \sum_{m_{i}, r_{i}, v, w} \int_{\mathbb{R}+i \epsilon-\frac{i k}{2}} \frac{d s}{2 i s+v}(-1)^{\sum_{i} m_{i}} q^{\frac{1}{2} \sum\left(m_{i}-\frac{1}{2}\right)^{2}} z^{\sum_{i}\left(m_{i}-\frac{1}{2}\right)} \\
q^{k w^{2}-v w} z^{N\left(2 w-\frac{v}{k}\right)}(q \bar{q})^{\frac{v^{2}}{4 k}+\frac{s^{2}}{k}}(q \bar{q})^{i s+\frac{v}{2}} z^{N} q^{k w-v} \delta_{\sum_{i}\left(r_{i}-m_{i}+1\right)-v+k w} \prod_{i=1}^{N} y_{i}^{\left(r_{i}-m_{i}+1\right)} S_{r_{i}} .
\end{gathered}
$$


The $z^{N}$ factor can be absorbed into each of the $z^{m_{i}-\frac{1}{2}}$ factors to get $z^{m_{i}+\frac{1}{2}}$; in order to match this with the expression in part $I$, we define

$$
n_{i}=m_{i}+1 \quad \text { and } \quad t_{i}=r_{i}+1
$$

We also use the identity $S_{t_{i}-1}=1-S_{-t_{i}}$ to obtain the following expression:

$$
\begin{gathered}
\chi_{N, I I}\left(\tau, \alpha, \beta_{i}\right)=\frac{1}{\pi \eta^{3 N}(\tau)} \sum_{n_{i}, t_{i}, v, w} \int_{\mathbb{R}+i \epsilon-\frac{i k}{2}} \frac{d s}{2 i s+v}(-1)^{\sum_{i} n_{i}} q^{\frac{1}{2} \sum\left(n_{i}-\frac{1}{2}\right)^{2}} z^{\sum_{i}\left(n_{i}-\frac{1}{2}\right)} \\
q^{k w^{2}-v w} z^{N\left(2 w-\frac{v}{k}\right)} q^{\sum_{i}\left(1-n_{i}\right)+k w-v}(q \bar{q})^{\frac{v^{2}}{4 k}+\frac{s^{2}}{k}+i s+\frac{v}{2}} \delta_{\sum_{i}\left(t_{i}-n_{i}+1\right)-(v-k w)} \\
\left(1-S_{\sum_{i}\left(1-n_{i}\right)+\sum_{j=1}^{N-1}} t_{j}-v+k w\right) \\
\prod_{j=1}^{N-1}\left(1-S_{-t_{j}}\right) \prod_{i=1}^{N} y_{i}^{\left(t_{i}-n_{i}+1\right)} .
\end{gathered}
$$

There are $2^{N}$ terms in the product of $N$ factors, each of the form $(1-S)$. We focus on the monomial arising from the product of $N S$ 's. Using the fact that the $q$-exponent can be absorbed by using the formula

$$
q^{r} S_{r}=S_{-r},
$$

we obtain the following expression (after relabeling $n_{i}$ as $m_{i}$ and $t_{i}$ as $r_{i}$ ):

$$
\begin{gathered}
\chi_{N, I I}^{S}\left(\tau, \alpha, \beta_{i}\right)=\frac{(-1)^{N}}{\pi \eta^{3 N}(\tau)} \sum_{m_{i}, r_{i}, v, w} \int_{\mathbb{R}+i \epsilon-\frac{i k}{2}} \frac{d s}{2 i s+v}(-1)^{\sum_{i} m_{i}} q^{\frac{1}{2} \sum\left(m_{i}-\frac{1}{2}\right)^{2}} z^{\sum_{i}\left(m_{i}-\frac{1}{2}\right)} \\
q^{k w^{2}-v w} z^{N\left(2 w-\frac{v}{k}\right)}(q \bar{q})^{\frac{v^{2}}{4 k}+\frac{s^{2}}{k}+i s+\frac{v}{2}} \delta_{\sum_{i=1}^{N}\left(r_{i}-m_{i}+1\right)-(v-k w)} \prod_{i=1}^{N} y_{i}^{\left(r_{i}-m_{i}+1\right)} S_{r_{i}} .
\end{gathered}
$$

We observe that the integrand above has the same form as that of $\chi_{N, I}$ in equation (3.13), the difference being the shifted contour of integration. Combining these two terms we obtain the following contour integral:

$$
\begin{aligned}
\chi_{N, h o l}\left(\tau, \alpha, \beta_{i}\right)= & \frac{(-1)^{N+1}}{\pi \eta^{3 N}(\tau)} \sum_{m_{i}, r_{i}, v, w}\left[\int_{\mathbb{R}+i \epsilon}-\int_{\mathbb{R}+i \epsilon-\frac{i k}{2}}\right] \frac{d s}{2 i s+v}(-1)^{\sum_{i} m_{i}} q^{\frac{1}{2} \sum\left(m_{i}-\frac{1}{2}\right)^{2}} z^{\sum_{i}\left(m_{i}-\frac{1}{2}\right)} \\
& (q \bar{q})^{i s+\frac{v}{2}} q^{k w^{2}-v w} z^{N\left(2 w-\frac{v}{k}\right)}(q \bar{q})^{\frac{v^{2}}{4 k}+\frac{s^{2}}{k}} \delta_{\sum_{i=1}^{N}\left(r_{i}-m_{i}+1\right)-(v-k w)} \prod_{i=1}^{N} y_{i}^{\left(r_{i}-m_{i}+1\right)} S_{r_{i}} .
\end{aligned}
$$

Due to the closed contour integral, with poles at $2 i s+v=0$, this combination is holomorphic, and corresponds to the contribution of right-moving ground states. The remaining $2^{N}-1$ terms make up the remainder term and will be dealt with in the next subsection. The contour integral can be done by picking up the poles. We have contributions whenever $v$ is an integer that lies between 0 and $-k+1$ :

$$
\begin{aligned}
\chi_{N, h o l}\left(\tau, \alpha, \beta_{i}\right)=\frac{(-1)^{N}}{\eta^{3 N}(\tau)} \sum_{m_{i}, r_{i}, w} \sum_{v=-k+1}^{0}(-1)^{\sum_{i} m_{i}} q^{\frac{1}{2} \sum\left(m_{i}-\frac{1}{2}\right)^{2}} z^{\sum_{i}\left(m_{i}-\frac{1}{2}\right)} \\
q^{k w^{2}-v w} z^{N\left(2 w-\frac{v}{k}\right)} \delta_{\sum\left(r_{i}-m_{i}+1\right)-(v-k w)} \prod_{i=1}^{N} y_{i}^{\left(r_{i}-m_{i}+1\right)} S_{r_{i}} .
\end{aligned}
$$


Now define the variable

$$
p_{i}=r_{i}-m_{i}+1
$$

an redefine the variable $m_{i}$ to $-m_{i}+1$ to obtain:

$$
\begin{aligned}
\chi_{N, h o l}\left(\tau, \alpha, \beta_{i}\right)=\frac{1}{\eta^{3 N}(\tau)} \sum_{m_{i}, p_{i}, w} \sum_{v=-k+1}^{0}(-1)^{\sum_{i} m_{i}} q^{\frac{1}{2} \sum\left(m_{i}-\frac{1}{2}\right)^{2}} z^{-\sum_{i}\left(m_{i}-\frac{1}{2}\right)} \\
q^{k w^{2}-v w} z^{N\left(2 w-\frac{v}{k}\right)} \delta_{\sum p_{i}-(v-k w)} \prod_{i=1}^{N} y_{i}^{p_{i}} S_{-m_{i}+p_{i}} .
\end{aligned}
$$

We now use repeatedly the formula (proven in appendix A.2):

$$
\frac{i \theta_{11}(\tau, \alpha)}{1-z q^{p}}=\sum_{m \in \mathbb{Z}}(-1)^{m} q^{\frac{1}{2}\left(m-\frac{1}{2}\right)^{2}} z^{m-\frac{1}{2}} S_{-m+p}
$$

and we see that the holomorphic piece can be written in the rather simple form

$$
\chi_{N, h o l}\left(\tau, \alpha, \beta_{i}\right)=\left(\frac{i \theta_{11}(\tau,-\alpha)}{\eta^{3}(\tau)}\right)^{N} \sum_{p_{i}, w} \sum_{v=-k+1}^{0} q^{k w^{2}-v w} z^{N\left(2 w-\frac{v}{k}\right)} \delta_{\sum p_{i}-(v-k w)} \prod_{i} \frac{y_{i}^{p_{i}}}{1-z^{-1} q^{p_{i}}} .
$$

We thus have that a purely holomorphic piece arises from the difference of contour integrals lying on the real and the shifted real axis.

Another form of the holomorphic state sum will be useful later on. We obtain it as follows. We first flip the sign of $v$ and $w$ in the equation (3.26) above. We split the $v$ summation into $N$ pieces, each of which goes from 0 to $M-1$. We put $v=V+j M$. Then, we obtain

$$
\begin{aligned}
\chi_{N, h o l}\left(\tau, \alpha, \beta_{i}\right)=\left(\frac{i \theta_{11}(\tau,-\alpha)}{\eta^{3}(\tau)}\right)^{N} & \sum_{p_{i}, w} \sum_{V=0}^{M-1} q^{k w^{2}-V w} z^{-N\left(2 w-\frac{V}{k}\right)} \\
& \times \sum_{j=0}^{N-1} q^{-j M w} z^{j} \delta_{\sum p_{i}+V-k w+j M} \prod_{i} \frac{y_{i}^{p_{i}}}{1-z^{-1} q^{p_{i}}} .
\end{aligned}
$$

\subsection{Further analysis of the holomorphic part}

In this subsection we analyze the character decomposition of the holomorphic part of the partition function, present a contour integral representation, and argue that the Witten index equals one.

\subsubsection{Character decomposition}

We wish to read the holomorphic part of the elliptic genus as a sum over characters. In order to facilitate this, we write the argument of the delta function as follows:

$$
\sum_{i=1}^{N} p_{i}-v+N M w=\sum_{i=1}^{N}\left(p_{i}+M w\right)-v
$$


Now we define $r_{i}=p_{i}+M w$ such that we can write the holomorphic part as

$$
\chi_{N, h o l}\left(\tau, \alpha, \beta_{i}\right)=\left(\frac{i \theta_{11}(\tau,-\alpha)}{\eta^{3}(\tau)}\right)^{N} \sum_{r_{i}, w} \sum_{v=-k+1}^{0} q^{k w^{2}-v w} z^{N\left(2 w-\frac{v}{k}\right)} \delta_{\sum r_{i}-v} \prod_{i} \frac{y_{i}^{r_{i}-M w}}{1-z^{-1} q^{r_{i}-M w}} .
$$

Let's proceed to exhibit the state space sum. To connect the holomorphic contribution in equation (3.29) to Ramond sector characters, we will reason in terms of a direct sum of $N$ $\mathcal{N}=2$ superconformal algebras with central charge $c_{f}=3(1+2 N / k)=3(1+2 / M)$. In such a factor model, we start out with a ground state character at $R$-charge $Q_{R}$ :

$$
\chi\left(Q_{R} ; q, z\right)=z^{Q_{R}-\frac{1}{2}} \frac{1}{1-z^{-1}} \frac{i \theta_{11}(\tau,-\alpha)}{\eta^{3}(\tau)} .
$$

If we spectrally flow the ground state representation by $-r$ units we find:

$$
\chi\left(Q_{R} ;-r ; q, z\right)=q^{\left(\frac{c_{f}}{6}-\frac{1}{2}\right) r^{2}} z^{-\left(\frac{c_{f}}{3}-1\right) r} z^{Q_{R}-\frac{1}{2}} q^{\left(-Q_{R}+\frac{1}{2}\right) r} \frac{1}{1-z^{-1} q^{r}} \frac{i \theta_{11}(\tau,-\alpha)}{\eta^{3}(\tau)} .
$$

We conclude that if we flow by $-r=\left(-Q_{R}+1 / 2\right) M$ units the character of the ensuing representation reads:

$$
\chi\left(Q_{R} ;-\left(Q_{R}-1 / 2\right) M ; q, z\right)=z^{-r N / k} \frac{1}{1-z^{-1} q^{r}} \frac{i \theta_{11}(\tau,-\alpha)}{\eta^{3}(\tau)} .
$$

We now consider a tensor product representation of the direct sum of the $\mathcal{N}=2$ superconformal algebras. The direct sum is an $\mathcal{N}=2$ superconformal algebra with central charge $c=3 N(1+2 / M)$. It has a character which is the product of the characters of the factor modules. Thus, if we multiply $N$ of the characters we just constructed, with spectral flow quantum numbers $r_{i}$ we find:

$$
\chi_{\otimes}\left(r_{i} ; q, z\right)=\left(\frac{i \theta_{11}(\tau,-\alpha)}{\eta^{3}(\tau)}\right)^{N} z^{-\sum_{i} r_{i} N / k} \prod_{i=1}^{N} \frac{1}{1-z^{-1} q^{r_{i}}} .
$$

Suppose now that we only allow for representations that have a sum of individual spectral flow quantum numbers (and therefore $R$-charges) which is equal to $v$, namely, $\sum_{i=1}^{N} r_{i}=v$. We sum over $v$ from 0 to $-k+1$. We then find the characters:

$$
\chi_{\otimes, \oplus}\left(v, r_{i} ; q, z\right)=\sum_{v=-k+1}^{0}\left(\frac{i \theta_{11}(\tau,-\alpha)}{\eta^{3}(\tau)}\right)^{N} z^{-N v / k} \delta_{\sum r_{i}-v} \prod_{i=1}^{N} \frac{y_{i}^{r_{i}}}{1-z^{-1} q^{r_{i}}} .
$$

We have also dressed the characters with global U(1) charges associated to each of the factor models. There is one more step to perform in order to obtain the characters of the modules featuring in the holomorphic contribution to our path integral real Jacobi form. In the direct $\operatorname{sum} \mathcal{N}=2$ superconformal algebra, we further spectrally flow by $w M$ units. Taking into account that the relevant charge is now the total central charge, and keeping track of the exponents of $q$ and $z$ carefully, one then obtains the characters:

$$
\chi_{\text {fin }}\left(w, v, r_{i} ; q, z\right)=\sum_{w \in \mathbb{Z}} q^{k w^{2}-v w} z^{2 N w} \sum_{v=-k+1}^{0}\left(\frac{i \theta_{11}(\tau,-\alpha)}{\eta^{3}(\tau)}\right)^{N} z^{-N v / k} \delta_{\sum r_{i}-v} \prod_{i=1}^{N} \frac{y_{i}^{r_{i}-M w}}{1-z q^{r_{i}-M w}} .
$$


Note that under spectral flow, the angular momentum of the state changes, since the Rcharge not only depends on fermion number but also on the angular momentum as can be seen by inspecting the exact $N=2$ superconformal algebra generators. Summing over all $r_{i}$ subject to the delta function constraint we note that this is equal to the holomorphic piece written in equation (3.29). We have thus established the full character decomposition of the holomorphic piece.

\subsubsection{A contour integral representation}

So far we have written out the holomorphic piece of the elliptic genus as a constrained sum over characters. It is also possible to write it as a contour integral; the final expression is similar to the integral expressions for the Appell-Lerch sums [17]. We begin by writing the delta function in equation (3.26) as

$$
\delta_{\sum p_{i}-(v-k w)}=\frac{1}{2 \pi i} \oint \frac{d x}{x} x^{\sum p_{i}-(v-k w)} .
$$

This unconstrains the $p_{i}$ variables which, in turn, allows us to do the $p_{i}$ summation. We obtain

$$
\chi_{N, h o l}\left(\tau, \alpha, \beta_{i}\right)=\frac{1}{2 \pi i} \oint \frac{d x}{x}\left(\frac{i \theta_{11}(\tau,-\alpha)}{\eta^{3}(\tau)}\right)^{N} \sum_{p_{i}, w} \sum_{v=-k+1}^{0} q^{k w^{2}-v w} z^{N\left(2 w-\frac{v}{k}\right)} x^{k w-v} \prod_{i} \frac{\left(x y_{i}\right)^{p_{i}}}{1-z^{-1} q^{p_{i}}} .
$$

Now, each of the $p_{i}$ summations can be done using the formula [28]

$$
\sum_{p} \frac{x^{p}}{1-z q^{p}}=\frac{i \theta_{11}(\tau, \alpha+\gamma) \eta^{3}(\tau)}{i \theta_{11}(\tau, \gamma) i \theta_{11}(\tau, \alpha)}
$$

Here, $x=e^{2 \pi i \gamma}$ and $z=e^{2 \pi i \alpha}$. Using this identity in the formula for the holomorphic part we get

$$
\chi_{N, h o l}\left(\tau, \alpha, \beta_{i}\right)=\frac{1}{2 \pi i} \sum_{w \in \mathbb{Z}} \sum_{v=-k+1}^{0} \oint \frac{d x}{x} q^{k w^{2}-v w} z^{N\left(2 w-\frac{v}{k}\right)} x^{k w-v} \prod_{i}\left[\frac{\theta_{11}\left(\tau, \gamma+\beta_{i}-\alpha\right)}{\theta_{11}\left(\tau, \gamma+\beta_{i}\right)}\right] .
$$

We also use the definition of the level $k$ theta function

$$
\Theta_{k, v}(\tau, \alpha)=\sum_{j \in \mathbb{Z}+\frac{v}{2 k}} q^{k j^{2}} z^{k j}
$$

to finally write the holomorphic part as the contour integral:

$$
\chi_{N, h o l}\left(\tau, \alpha, \beta_{i}\right)=\frac{1}{2 \pi i} \oint \frac{d x}{x} \sum_{v=0}^{k-1} q^{-\frac{v^{2}}{4 k}} x^{\frac{v}{2}} \Theta_{k, v}\left(\tau, \frac{2 N \alpha}{k}+\gamma\right) \prod_{i}\left[\frac{\theta_{11}\left(\tau, \gamma+\beta_{i}-\alpha\right)}{\theta_{11}\left(\tau, \gamma+\beta_{i}\right)}\right] .
$$

This is close to expressions for truly holomorphic elliptic genera arising from gauged linear sigma models as well as ordinary two-dimensional gauge theories. As such it seemingly allows for an interpretation in terms of charged fields, a zero mode, and a U(1) gauge field. While this is true, it is not the whole story since modular covariance necessitates a completion. 


\subsubsection{The Witten index}

To compute the Witten index of the model, it is easiest to take the $\alpha=0$ path integral expression in equation (2.2). A short calculation then gives a Witten index equal to one for all values of $N$ and $k$.

\subsection{The modular completion of the mock modular form}

In this subsection, we return to analyzing the remainder term of the path integral expression, namely the modular completion of the mock modular form. We wish to massage this term into a compact form, and then interpret it.

\subsubsection{A succinct expression}

Recall that we obtained the holomorphic piece by combining one term out of $2^{N}$ from (3.18) with the expression in (3.13). We are therefore left with multiple non-holomorphic pieces:

$$
\begin{aligned}
\chi_{N}^{r e m}\left(\tau, \alpha, \beta_{i}\right)=\frac{1}{\pi \eta^{3 N}(\tau)} \sum_{m_{i}, r_{i}, v, w} \int_{\mathbb{R}+i \epsilon-\frac{i k}{2}} \frac{d s}{2 i s+v}(-1)^{\sum_{i} m_{i}} q^{\frac{1}{2} \sum\left(m_{i}-\frac{1}{2}\right)^{2}} z^{\sum_{i}\left(m_{i}-\frac{1}{2}\right)} q^{k w^{2}-v w} z^{N\left(2 w-\frac{v}{k}\right)} \\
(q \bar{q})^{\frac{v^{2}}{4 k}+\frac{s^{2}}{k}+i s+\frac{v}{2}} q^{\sum_{i}\left(1-m_{i}\right)-v+k w} \delta_{\sum_{i}\left(r_{i}-m_{i}+1\right)-(v-k w)} \\
{\left[\prod_{i=1}^{N} y_{i}^{\left(r_{i}-m_{i}+1\right)} S_{r_{i}-1}-(-1)^{N} \prod_{i=1}^{N} y_{i}^{\left(r_{i}-m_{i}+1\right)} S_{-r_{i}}\right] . }
\end{aligned}
$$

Here we have written the remainder as the difference of the equation (3.18) and the piece we take out from it, namely (3.20). The contour is now at the shifted location in the $s$-plane. We undo the shifts in order to write the integral over the real axis with real exponent:

$$
s \rightarrow s-\frac{i k}{2} \quad v \rightarrow v-k \quad w \rightarrow w-1 .
$$

Again, the combinations $2 i s+v$ and $v-k w$ are left invariant under this shift. Also, note that the imaginary part in the $(q \bar{q})$ exponent vanishes. Defining $n_{i}=m_{i}-1$, one can write this as

$$
\begin{array}{r}
\chi_{N}^{r e m}\left(\tau, \alpha, \beta_{i}\right)=\frac{(-1)^{N}}{\pi \eta^{3 N}(\tau)} \sum_{n_{i}, r_{i}, v, w} \int_{\mathbb{R}+i \epsilon} \frac{d s}{2 i s+v}(-1)^{\sum_{i} n_{i}} q^{\frac{1}{2} \sum\left(n_{i}-\frac{1}{2}\right)^{2}} z^{\sum_{i}\left(n_{i}-\frac{1}{2}\right)} q^{k w^{2}-v w} z^{N\left(2 w-\frac{v}{k}\right)} \\
(q \bar{q})^{\frac{v^{2}}{4 k}+\frac{s^{2}}{k}} \delta_{\sum_{i}\left(r_{i}-n_{i}\right)-(v-k w)}\left[\prod_{i=1}^{N} y_{i}^{\left(r_{i}-n_{i}\right)} S_{r_{i}-1}-(-1)^{N} \prod_{i=1}^{N} y_{i}^{\left(r_{i}-n_{i}\right)} S_{-r_{i}}\right] .
\end{array}
$$

As for the holomorphic part, we redefine the summation variables by setting $p_{i}=r_{i}-n_{i}$ and we once again use the identity (3.25) to simplify the resulting expression to obtain

$$
\begin{aligned}
\chi_{N}^{r e m}\left(\tau, \alpha, \beta_{i}\right)=\frac{1}{\pi \eta^{3 N}(\tau)} \sum_{v, w} q^{k w^{2}-v w} z^{N\left(2 w-\frac{v}{k}\right)} \int_{\mathbb{R}+i \epsilon} \frac{d s}{2 i s+v}(q \bar{q})^{\frac{v^{2}}{4 k}+\frac{s^{2}}{k}} \\
\sum_{p_{i} \in \mathbb{Z}} \delta_{\sum_{i} p_{i}-v+k w}\left[\prod_{i=1}^{N} \frac{i \theta_{11}(\tau,-\alpha) y_{i}^{p_{i}}}{1-z^{-1} q^{p_{i}}}-\prod_{i=1}^{N} \frac{i \theta_{11}(\tau, \alpha) y_{i}^{p_{i}}}{1-z q^{-p_{i}}}\right] .
\end{aligned}
$$

Using the symmetry property of the theta function and the delta function constraint, this can be simplified to

$$
\chi_{N}^{r e m}\left(\tau, \alpha, \beta_{i}\right)=\left(\frac{i \theta_{11}(\tau,-\alpha)}{\pi \eta^{3}(\tau)}\right)^{N} \sum_{v, w} q^{k w^{2}-v w} z^{N\left(2 w-\frac{v}{k}\right)}\left(1-z^{-N} q^{v-k w}\right)
$$




$$
\int_{\mathbb{R}+i \epsilon} \frac{d s}{2 i s+v}(q \bar{q})^{\frac{v^{2}}{4 k}+\frac{s^{2}}{k}} \sum_{p_{i} \in \mathbb{Z}} \delta_{\sum_{i} p_{i}-v+k w} \prod_{i=1}^{N} \frac{y_{i}^{p_{i}}}{\left(1-z^{-1} q^{p_{i}}\right)} .
$$

An alternate way to write the remainder term is in terms of the variables $n$ and $w$ by writing $v=n+k w$. The exponent of $q$ can be simplified by completing the square and we find the following expression for the remainder:

$$
\begin{aligned}
& \chi_{N}^{r e m}\left(\tau, \alpha, \beta_{i}\right)=\left(\frac{i \theta_{11}(\tau,-\alpha)}{\pi \eta^{3}(\tau)}\right)^{N} \sum_{n, w} z^{N \frac{(k w-n)}{k}}\left(1-z^{-N} q^{n}\right) \\
& \quad \times \int_{\mathbb{R}+i \epsilon} \frac{d s}{2 i s+n+k w} q^{\frac{(n-k w)^{2}}{4 k}+\frac{s^{2}}{k}} \bar{q}^{\frac{(n+k w)^{2}}{4 k}+\frac{s^{2}}{k}} \sum_{p_{i} \in \mathbb{Z}} \delta_{\sum_{i} p_{i}-n} \prod_{i=1}^{N} \frac{y_{i}^{p_{i}}}{\left(1-z^{-1} q^{p_{i}}\right)} .
\end{aligned}
$$

Recovering the cigar answer. For the $N=1$ case we should recover the known cigar answer; for the holomorphic piece we obtain

$$
\chi_{1, h o l}\left(\tau, \alpha, \beta_{i}\right)=\frac{i \theta_{11}(\tau,-\alpha)}{\eta^{3}(\tau)} \sum_{w \in \mathbb{Z}} \sum_{v=-k+1}^{0} q^{k w^{2}-v w} z^{2 w-\frac{v}{k}} \frac{y^{v-k w}}{1-z^{-1} q^{v-k w}} .
$$

Here we have explicitly solved for the $p_{1}$ variable using the delta function. Similarly, setting $N=1$ in the remainder piece, we find:

$$
\chi_{1}^{r e m}\left(\tau, \alpha, \beta_{i}\right)=\frac{i \theta_{11}(\tau,-\alpha)}{\pi \eta^{3}(\tau)} \sum_{v, w \in \mathbb{Z}} q^{k w^{2}-v w} z^{2 w-\frac{v}{k}} \int_{\mathbb{R}+i \epsilon} \frac{d s}{2 i s+v}(q \bar{q})^{\frac{v^{2}}{4 k}+\frac{s^{2}}{k}} y^{v-k w} .
$$

We now flip the sign of $v$ and $w$ in both terms and also flip the sign of $s$ in the remainder term. We observe that compared to the formulas in [10], both $z$ and $y$ appear with inverse powers. The charge conjugation symmetry in (2.14) implies $^{2}$

$$
\chi_{1}(\tau,-\alpha,-\beta)=\chi_{1}(\tau, \alpha, \beta),
$$

and we recover the elliptic genus obtained in [10].

\subsubsection{Observations on the modular completion}

We make a few observations on the modular completion.

The shadow. Since we have the explicit modular completion, it is straightforward to calculate the shadow, which is obtained as the $\partial_{\bar{\tau}}$-derivative of the elliptic genus. The only dependence comes from the integral $I$ in the last line of equation (3.47). We obtain

$$
\begin{aligned}
I & =\int_{\mathbb{R}+i \epsilon} \frac{d s}{2 i s+v}(q \bar{q})^{\frac{v^{2}}{4 k}+\frac{s^{2}}{k}} . \\
\partial_{\bar{\tau}} I & =-\frac{i \pi}{2 k} \int d s(v-2 i s)(q \bar{q})^{\frac{v^{2}}{4 k}+\frac{s^{2}}{k}}
\end{aligned}
$$

\footnotetext{
${ }^{2}$ For the $y=1$ case, this was explicitly shown in [18] by studying the transformation properties of the holomorphic and remainder pieces separately. In particular, the analysis incorporates a careful treatment of the $\epsilon$ contour prescription.
} 


$$
=-\frac{i \pi v}{4 \sqrt{k \tau_{2}}}(q \bar{q})^{\frac{v^{2}}{4 k}} .
$$

Substituting this into the $\partial_{\bar{\tau}}$-derivative of the elliptic genus we obtain

$$
\begin{aligned}
\partial_{\bar{\tau}} \chi_{L_{N}}\left(\tau, \alpha, \beta_{i}\right)=-\frac{i \pi}{2} \sqrt{\frac{k}{\tau_{2}}} & \left(\frac{i \theta_{11}(\tau,-\alpha)}{\pi \eta^{3}(\tau)}\right)^{N} \sum_{v, w \in \mathbb{Z}} q^{k w^{2}-v w+\frac{v^{2}}{4 k}} z^{N\left(2 w-\frac{v}{k}\right)} \frac{v}{2 k} \bar{q}^{\frac{v^{2}}{4 k}} \\
& \times\left(1-z^{-N} q^{v-k w}\right) \sum_{p_{i} \in \mathbb{Z}} \delta_{\sum_{i} p_{i}-v+k w} \prod_{i=1}^{N} \frac{y_{i}^{p_{i}}}{\left(1-z^{-1} q^{p_{i}}\right)} .
\end{aligned}
$$

Writing this in terms of $n=v-k w$, we find that

$$
\begin{array}{r}
\partial_{\bar{\tau}} \chi_{L_{N}}\left(\tau, \alpha, \beta_{i}\right)=-\frac{i \pi}{2} \sqrt{\frac{k}{\tau_{2}}}\left(\frac{i \theta_{11}(\tau,-\alpha)}{\pi \eta^{3}(\tau)}\right)^{N} \sum_{n, w \in \mathbb{Z}} \frac{n+k w}{2 k} z^{-N\left(\frac{n-k w}{k}\right)} q^{\frac{(n-k w)^{2}}{4 k}} \bar{q}^{\frac{(n+k w)^{2}}{4 k}} \\
\times\left(1-z^{-N} q^{-n}\right) \sum_{p_{i} \in \mathbb{Z}} \delta_{\sum_{i} p_{i}-n} \prod_{i=1}^{N} \frac{y_{i}^{p_{i}}}{\left(1-z^{-1} q^{p_{i}}\right)}
\end{array}
$$

For $N>1$ we note that the sums over $n+k w$ and $n-k w$ are coupled. This has the following consequence. In the examples with $N=1$ studied in $[6,10]$, the shadow is a modular form of given weight $[7,8,30]$. In these instances it is a (finite) sum over products of level $k$ theta functions [18]. As can be seen from equation (3.53) this is not the case for $N>1$. Again, as in the example of elliptic genera of (orbifolds of) tensor product conformal field theories [18], we see that in physical applications the set of mock modular forms, completions and shadows that can arise is richer than the class that is at present under good mathemical control [30].

A character decomposition. The character decomposition of the remainder term is harder to understand. We have many more degrees of freedom than those represented by the overall $\mathcal{N}=2$ superconformal algebra and thus, we expect an infinite sum and integral over individual representations of the overall algebra. In contrast with the holomorphic contribution, naively taking tensor products of factor representations does not give rise to the characters that we find. One could identify all primary states with respect to the overall $\mathcal{N}=2$ superconformal algebra (only) in the remainder term, but that is tedious.

Rather, we can show indirectly that the expression must permit and $\mathcal{N}=2$ superconformal interpretation, as follows. Under modular S-transformation, the sum of the holomorphic and non-holomorphic terms transforms covariantly. Moreover, we know that the characters we identified in the holomorphic part will transform into both discrete and integrals over continuous characters [19-21]. The latter contributions must conspire to give a term of the form of the remainder term. The remainder term must then permit an interpretation as an (albeit complicated) sum and integral over discrete and continuous characters.

Summary. We thus have gained insight into the decomposition of our path integral into a holomorphic state space sum, the term that modularly completes the mock Jacobi form to a real Jacobi form with matrix index, and the corresponding shadow. We now turn 
to the proposal for the identification of the conformal field theory whose elliptic genus we have analyzed hitherto.

\section{Asymptotically linear dilaton conformal field theory}

In this section, we wish to argue that the path integral expressions we have obtained correspond to the elliptic genera of a family of conformal field theories that are natural generalizations of the cigar to higher dimensions. We give evidence for this in terms of a gauged linear sigma model, a counting of bound states, as well as in terms of the asymptotic geometries of these models.

\subsection{Asymptotically linear dilaton target spaces}

The backgrounds we consider are non-linear sigma models with target spaces that are asymptotically linear dilaton theories in $d=2 N$ dimensions [12-14]. The background metric and dilaton that solve the string beta function equations to first order in $\alpha^{\prime}$ are [12]:

$$
\begin{aligned}
d s_{K K L}^{2} & =\frac{g_{N}(Y)}{2} d Y^{2}+\frac{2}{N^{2} g_{N}(Y)}\left(d \psi+N A_{F S}\right)^{2}+2 Y d s_{\mathbb{C P}^{N-1}}^{2} \\
\Phi & =-\frac{N Y}{k}
\end{aligned}
$$

Here $Y$ is a non-compact radial coordinate; $\psi$ is a periodic variable with period $2 \pi N$ and the circle it parameterizes is fibered over the complex projective space $\mathbb{C P}^{N-1}$. The connection $A_{F S}$ is the Fubini-Study connection one-form whose differential is the Kähler form on $\mathbb{C P}^{N-1}$. The background is Kähler and has a $\mathrm{U}(N)$ isometry group. More details about the geometry and its construction can be found in $[11,12]$.

For the case $N=1$, the background is equivalent to the cigar geometry. In this case it is known that all $\alpha^{\prime}$ corrections can be taken into account by rewriting the model as a gauged Wess-Zumino-Witten model $\mathrm{SL}(2, \mathbb{R}) / \mathrm{U}(1)$ [22-24]. For $N>1$, a direct exact conformal field theory description of the conformal fixed point is unknown, but certain properties of the infrared theory have been derived. First of all, we know a gauged linear sigma model description in the ultraviolet whose infrared fixed point corresponds to the non-linear sigma model described above $[13,14]$. Secondly, this ultraviolet description allows for the exact calculation of the conformal field theory central charge:

$$
c=3 N\left(1+\frac{2 N}{k}\right) .
$$

This precisely coincides with the central charge we have obtained by studying the modular properties of the Jacobi forms in equation (2.2).

We note that it is an outstanding problem to derive the elliptic genera of these conformal field theories from first principles. A step in this direction was to obtain the spectrum of particular fundamental string bound states in these higher dimensional backgrounds. These states are labeled by (asymptotic) momentum and winding along the $\psi$ direction. In [11] their degeneracy was calculated by mapping the problem of finding wound bound 
states to counting the ground states of a supersymmetric quantum mechanics obtained by Scherk-Schwarz reduction of the sigma model action along the $\psi$ circle.

In what follows, we provide some evidence that the path integral expressions we have obtained correspond to the elliptic genera of this family of conformal field theories, labeled by the complex dimension $N$ and asymptotic radius $R=\sqrt{k \alpha^{\prime}}$. To that end, we first argue that the gauged linear sigma model description has the salient features to give rise to our path integral result. Secondly, we show that the counting of wound bound states is incorporated in our elliptic genus. Thirdly, we will argue that the remainder term is consistent with the asymptotic geometry of our models.

\subsection{The gauged linear sigma model path integral}

In this section we give heuristic arguments in support of our proposal by recalling that there is a gauged linear sigma model (GLSM) description of the backgrounds in equation (4.1) [13, 14]. In this description we consider a $\mathcal{N}=(2,2)$ supersymmetric $\mathrm{U}(1)$ gauge theory in two dimensions with $N$ chiral superfields $\Phi_{i}$, a U(1) vector multiplet $V$ and a Stückelberg superfield $P$ whose imaginary part transforms additively under gauge transformations. The superspace action for the gauged linear sigma model is given by [13]:

$$
S=\frac{1}{2 \pi} \int d^{2} x d^{4} \theta\left[\sum_{i=1}^{N} \bar{\Phi}_{i} e^{V} \Phi_{i}+\frac{k}{4}(P+\bar{P}+V)^{2}-\frac{1}{2 e^{2}} \bar{\Sigma} \Sigma\right] .
$$

Here, $\Sigma$ is a twisted chiral superfield derived from the vector multiplet. The fields $\Phi_{i}$ carry charge 1 under the $\mathrm{U}(1)$ gauge group. The fermionic components of the chiral superfields carry unit $R$-charge under the classical vector and axial $R$-symmetries of the $\mathcal{N}=(2,2)$ supersymmetric Lagrangian. The imaginary part of the $P$-field is a compact boson and its zero mode acquires $R$-charge via the chiral anomaly [13].

In $[13,14]$ it is shown in detail that the gauged linear sigma model described by the action (4.3) flows in the infrared to a non-linear sigma-model with the target geometry (4.1). For the case of a single chiral superfield $\Phi$, if we write the bosonic component $\phi=\rho e^{i \theta}$, it can be shown that the gauge invariant combination $\operatorname{Im}(P)-\theta$ is identified with the coordinate $\psi$ in the cigar geometry. Similarly, $\rho$ is identified (up to a coordinate transformation), with the radial coordiate $Y$ of the cigar. For the case of multiple chiral fields $\Phi_{i}$, the sum of the phases of the individual fields and the imaginary part of $P$ can be identified with the angle $\psi$, while the other directions will make up the $\mathbb{C P}^{N-1}$ and the radial direction.

We will now try to use this linear sigma model description to motivate our path integral expression (2.2). As explained in the introduction, the elliptic genus of the cigar has been derived from first principles by using a gauged Wess-Zumino-Witten description [10]. We reproduce the expression for convenience:

$$
\chi_{\cos }(\tau, \alpha)=k \int_{0}^{1} d s_{1} d s_{2} \sum_{m, w}\left[\frac{\theta_{11}\left(\tau, s_{1} \tau+s_{2}-\alpha\right)}{\theta_{11}\left(\tau, s_{1} \tau+s_{2}\right)} e^{2 \pi i \alpha w}\right] e^{-\frac{\pi k}{\tau_{2}}\left|m+w \tau+\left(s_{1} \tau+s_{2}+\frac{\alpha}{k}\right)\right|^{2}}
$$

Here we have shifted the variable $u=s_{1} \tau+s_{2}$ by $\frac{\alpha}{k}$ in order to write the above expression. ${ }^{3}$ We now heuristically identify the ratio of theta functions appearing in the above

\footnotetext{
${ }^{3}$ We are grateful to Nima Doroud and Jaume Gomis for clarifying this point.
} 
expression along with the phase factor as coming from the single chiral multiplet and the non-holomorphic piece as arising from the (charged) compact zero mode of the imaginary part of the $P$-field.

A guiding principle in writing down the expressions for the elliptic genera for general $N$ is the gauged linear sigma model description of these models. Indeed, we include $N$ thetafunction ratios for the $N$ chiral superfields $\Phi_{i}$ in the model [5], and recall that although the action for the superfield $P$ is unchanged, the appropriate $R$-charge of the $\operatorname{Im}(P)$-field in the infrared is now multiplied by a factor of $N$ [13]. We then see that these are the appropriate ingredients to give rise to our path integral expression in (2.2), where we have shifted the $u$ variable by $\frac{N \alpha}{k}$ :

$$
\chi_{N}\left(\tau, \alpha, \beta_{i}\right)=k \int_{0}^{1} d s_{1} d s_{2} \sum_{m, w} \prod_{i=1}^{N}\left[\frac{\theta_{11}\left(\tau, s_{1} \tau+s_{2}-\alpha-\frac{N \alpha}{k}+\beta_{i}\right)}{\theta_{11}\left(\tau, s_{1} \tau+s_{2}-\frac{N \alpha}{k}+\beta_{i}\right)}\right] e^{2 \pi i N \alpha w} e^{-\frac{\pi k}{\tau_{2}}\left|m+w \tau+\left(s_{1} \tau+s_{2}\right)\right|^{2}} .
$$

Here we have introduced the chemical potentials $\beta_{i}$ associated to the phase rotations of the fields $\Phi_{i}$, in order to match the general proposal in (2.2). Our arguments have been heuristic and a detailed analysis of the localization mechanism is necessary since our model falls outside the class of models studied for instance in [25-27]. This is because, as shown in [13], the action of the $P$-field in the gauged linear sigma model is not $Q$-exact. Consequently it is necessary to redo the localization analysis in the gauged linear sigma-model in the presence of the Stückelberg superfield $P .{ }^{4}$

\subsection{The count of wound bound states}

We have given some arguments in support of our path integral expression for the elliptic genus. We now provide more detailed evidence, by comparing contributions to our proposed elliptic genus with contributions from wound bound states identified in [11].

\subsubsection{Counting bound states from supersymmetric quantum mechanics}

Let us briefly review the index result we obtained in [11], in terms of the variables used in this paper. In that work, we started from a supersymmetric sigma model in $1+1$ dimensions with target space given by equation (4.1) and derived a supersymmetric quantum mechanical model in $0+1$ dimensions by Scherk-Schwarz reduction of the sigma model on (4.1) along the $\psi$ direction. Effectively it gave rise to a supersymmetric quantum mechanics with a gauge field given by

$$
A=\frac{2 w}{N g_{N}(Y)}\left(d \psi+N A_{F S}\right)
$$

The string winds around the $\psi$ circle $w$ times. We then calculated the (signed) number of zero mode solutions to the Dirac equation in the background (4.1), supplemented with the gauge field in (4.6). The answer to this problem can be summarized as follows (e.g. for

\footnotetext{
${ }^{4}$ Work in progress with Nima Doroud.
} 
even $N)[11]:^{5}$

$$
Z_{N}^{\prime}\left(y_{1}, y_{2}\right)=\left[\sum_{w<0} \sum_{n=N / 2}^{-k w}-\sum_{w>0} \sum_{n=-k w+1}^{-N / 2}\right] D(n-N / 2+1, N) y_{1}^{n} y_{2}^{w}
$$

where

$$
D(n-N / 2+1, N)=\left(\begin{array}{c}
n+N / 2-1 \\
N-1
\end{array}\right)
$$

The degeneracy factor depends on the momentum $n$ and is independent of the winding number $w$. It arises from the degeneracy of Landau levels in a quantum Hall system on the $\mathbb{C P} \mathbb{P}^{N-1}$ section, as described in [11]. Here, $y_{1}$ and $y_{2}$ are dummy variables that are chemical potentials for momentum and winding respectively. We wish to rewrite this result as a double signed sum over wedges. To that end, we wish to perform the sum over $n$ first. We write:

$$
Z_{N}^{\prime}\left(y_{1}, y_{2}\right)=\left[\sum_{n \geq N / 2} \sum_{n+k w \leq 0}-\sum_{n \leq-N / 2} \sum_{n+k w>0}\right] D(n-N / 2+1, N) y_{1}^{n} y_{2}^{w} .
$$

The answer above is obtained by counting zero modes of a space-time fermion; consequently it is a calculation in e.g. the $N S-R$ sector on the worldsheet. In order to compare with our elliptic genus calculation, we have to write the answer in the $R$ - $R$ sector in which we worked up to now. Left-moving spectral flow will shift the momentum $n$ by $\frac{N}{2}$ and will leave the right-moving momentum invariant. We then obtain:

$$
Z_{N}\left(y_{1}, y_{2}\right)=\left[\sum_{n \geq 0} \sum_{n+k w \leq 0}-\sum_{n \leq-N} \sum_{n+k w>0}\right] D(n+1, N) y_{1}^{n} y_{2}^{w} .
$$

To supply suitable weights that depend on the $q$ and $z$ variables keeping track of conformal dimension and left-moving $R$-charge, we perform a few calculations. Since the holomorphic contribution to the elliptic genus arises from right moving ground states, the modes we look for have $\bar{L}_{0}=\frac{c}{24}$; thus, the exponent of $q$ is equal to

$$
L_{0}-\frac{c}{24}=L_{0}-\bar{L}_{0}
$$

The difference $L_{0}-\bar{L}_{0}$ is equal to the central charge of the super quantum mechanics; this, in turn, can be obtained by acting with the differential operator (see e.g. [29])

$$
Z=-i K^{\mu} \nabla_{\mu}-\frac{i}{2}\left(\nabla_{\mu} K_{\nu}\right) \Gamma^{\mu} \Gamma^{\nu}
$$

on the explicit solutions obtained in [11]. The differential operator depends on the Killing vector $K$ dual to the gauge field $A$ as well as on the covariant derivative $\nabla$ and gamma matrices $\Gamma$. The operator is the Lie derivative acting on spinors. The result of the evaluation is $L_{0}-\bar{L}_{0}=-n w$. This is as expected.

\footnotetext{
${ }^{5}$ Compared to [11], we normalized $n$ such that it is an integer, flipped its sign, used the fact that $D(-n-N / 2+1, N)=(-1)^{N-1} D(n-N / 2+1, N)$ as well as the property that the overall sign of $Z_{N}^{\prime}$ is a matter of convention, and picked a particular regularization (discussed in [11]).
} 
In order to obtain the exponent of $z$, it is necessary to know the R-current at the IR fixed point. As our guideline, we will use the $\mathcal{N}=2$ superconformal algebra in terms of the fields of the gauged linear sigma model [13]. We are only concerned with the contribution to the R-charge from the momentum and winding modes on the asymptotic circle direction. As discussed earlier, in the gauged linear sigma model, this circle direction is identified with the imaginary part of the $P$ superfield. The contribution to the R-charge from the $\operatorname{Im}(P)$ field can be read off from section 7 of $[13]$ to be ${ }^{6}$

$$
N\left(\frac{n-k w}{k}\right) \text {. }
$$

We believe this answer is also valid at the infrared fixed point. Putting these facts together we find the following expression as a contribution to the elliptic genus:

$$
Z_{N}(q, z)=\left[\sum_{n \geq 0} \sum_{n+k w \leq 0}-\sum_{n \leq-N} \sum_{n+k w>0}\right] D(n+1, N) q^{-n w} z^{\frac{N(n-k w)}{k}} .
$$

\subsubsection{Matching wound bound states}

In order to obtain the wound bound states of fundamental strings in the geometry (4.1), we wound a string on the asymptotic $\psi$-circle, and computed the number of bound states, given that neither oscillator excitations nor other winding numbers were turned on. We saw that these bound states carried left-moving conformal dimension equal to $-n w+c / 24$, which fixes the power of the modular parameter $q$ in the partition sum to be of the product form. We now identify the particular terms in the holomorphic part of the elliptic genus corresponding to these particular bound states. Recall that the elliptic genus is charge conjugation symmetric, as noted in equation (2.14). For easier comparison to the index result of [11], we will work with the charge conjugate holomorpic contribution:

$$
\chi_{N, h o l}\left(\tau, \alpha, \beta_{i}\right)=\left(\frac{i \theta_{11}(\tau, \alpha)}{\eta^{3}(\tau)}\right)^{N} \sum_{p_{i}, w} \sum_{v=-k+1}^{0} q^{k w^{2}-v w} z^{-N\left(2 w-\frac{v}{k}\right)} \delta_{\sum p_{i}-v+k w} \prod_{i} \frac{y_{i}^{p_{i}}}{1-z q^{p_{i}}} .
$$

To identify the relevant states in our partition sum, we recall the identification of the quantum number $v$ with the right-moving asymptotic momentum $n+k w$, such that the constraint reads

$$
\sum_{i=1}^{N} p_{i}=n
$$

In order to expand the denominators in the product, we assume that $|q|<|z|<1$. We further assume that all integers $p_{i}$ and $n$ are of the same sign. For $n \geq 0$, we find that the number of solutions for the integers $p_{i}$ is given by

$$
\left(\begin{array}{c}
n+N-1 \\
N-1
\end{array}\right)
$$

\footnotetext{
${ }^{6}$ It is important to recall that the $P$ field is not canonically normalized in the gauged linear sigma model action of [13].
} 
On the other hand, for $n<0$, the degeneracy is

$$
\left(\begin{array}{c}
-n-1 \\
N-1
\end{array}\right)
$$

up to an overall sign. These two expressions are related by a factor $(-1)^{N-1}$. The number of solutions therefore matches the degeneracy of the wound bound string ground states. Although we already identified the degeneracy, we still need to argue that we can freely sum over momentum and winding, since our holomorphic partition sum only contains a sum over the number $v$ in a particular range. To that end, we expand our partition sum using the assumption that either all $p_{i}$ are positive, or strictly negative. These two possibilities (out of $2^{N}$ ) lead to the terms:

$$
\begin{aligned}
& Z_{h o l, N}\left(\tau, \alpha, \beta_{i}\right)=\sum_{w \in \mathbb{Z}} \sum_{v=-k+1}^{0} z^{N v / k} q^{k w^{2}-v w} z^{-2 N w} \\
& \times\left[\sum_{p_{i} \geq 0} \sum_{w_{i} \geq 0} z^{\sum_{i} w_{i}} q^{\sum_{i} w_{i} p_{i}}+\cdots+(-1)^{N} \sum_{p_{i}<0} \sum_{w_{i} \geq 0} z^{-N-\sum_{i} w_{i}} q^{-\sum p_{i}} q^{-\sum_{i} w_{i} p_{i}}\right] \delta_{\sum p_{i}=n} \prod_{i=1}^{N} y_{i}^{p_{i}} .
\end{aligned}
$$

We have dropped the prefactors corresponding to oscillator excitations not captured by the supersymmetric quantum mechanics. We wish to think of $w_{i}$ as a winding number associated to the phase of the projective coordinate $\Phi_{i}$ of $\mathbb{C P}^{N-1}$. The strings wound in [11] wound only the overall $\psi$ coordinate, or in other words, each $\Phi_{i}$ phase an equal number of times. We therefore restrict to windings $w_{i}=r$ in the above sum and find:

$$
\begin{aligned}
& Z_{\text {restr }, \text { hol }, N}\left(\tau, \alpha, \beta_{i}\right)=\sum_{w \in \mathbb{Z}} \sum_{v=-k+1}^{0} q^{-n w} z^{-N w} z^{N n / k} \\
& {\left[\sum_{p_{i} \geq 0} \sum_{r \geq 0} z^{N r} q^{r n}+\cdots+(-1)^{N} \sum_{p_{i}<0} \sum_{r \geq 0} z^{-N(r+1)} q^{-(r+1) n}\right] \prod_{i=1}^{N} y_{i}^{p_{i}} \delta_{\sum p_{i}=n} .}
\end{aligned}
$$

In the summation term with all $p_{i}>0$, we introduce the new variable

$$
\tilde{w}=w-r
$$

in terms of which we see that the constraints $-(k-1) \leq v \leq 0$ and $n \geq 0$ as well as $r \geq 0$ map to the conditions $n+k \tilde{w} \leq 0$ and $n \geq 0$. The summation over the finite range for $v$ as well as the constraint $v=n+k w$ imposes a strong constraint on the sum over $w$. This constraint is removed through the shift by $r$, and the sum over the finite range of $v$ and the infinite range of $r$ is replaced by a summation over the integer $n+k \tilde{w}$. The sum over $n$ is then free, up to the constraint on its sign. Similarly in the last term, we consider the change of variables:

$$
\tilde{w}=w+r+1 .
$$

For these terms, the constraints become $n+k \tilde{w}>0$ and $n<0$. The sum over the integers $p_{i}$ subject to the constraint precisely reproduces the degeneracy factor, as mentioned earlier. The chemical potentials $y_{i}$ keep track of the origin of the degeneracy in the sum over 
$\mathrm{U}(1)^{N} \subset \mathrm{U}(N)$ angular momenta. If we put $y_{i}=1$, we find (after removing the tilde from the variable $\tilde{w})$ :

$$
Z_{r e s t r, h o l, N}(\tau, \alpha)=\left[\sum_{n \geq 0} \sum_{n+k w \leq 0}+\ldots-\sum_{n \leq-N} \sum_{n+k w>0}\right]\left(\begin{array}{c}
n+N-1 \\
N-1
\end{array}\right) q^{-n w} z^{N\left(\frac{n-k w}{k}\right)} .
$$

We have canceled the sign $(-1)^{N}$ against the factor $(-1)^{N-1}$ that arose when counting the number of solutions. We have found a precise agreement with equation (4.14).

It would be interesting to generalize the supersymmetric quantum mechanics that arose from Scherk-Schwarz reduction on the $\psi$-circle in [11] to the case where we allow for windings along other angular coordinates. We also expect these indices to be captured by our proposal for the elliptic genus.

\subsection{Features of the asymptotic geometry and susy algebra}

Finally, we turn to arguing that the remainder term is in accord with the asymptotic geometry of our model. Indeed, from the form of the modular completion, one can infer several things about the conformal field theory. For the case of the cigar (at $N=1$ ) [10], the remainder function in (3.49) can be interpreted as a sum over states in the cigar conformal field theory. One interprets the $s$-integration as an integral over the radial momentum of the states; in [10] it was shown that the measure of the $s$ integral is given by the spectral asymmetry between the bosons and fermions in the continuum sector of the conformal field theory. In [11] we argued the difference in density is fixed in terms of the asymptotic right-moving supercharge. The sum over $n$ and $w$ can be interpreted as the sum over momentum and winding modes on the asymptotic circle direction in the cigar geometry. The combination $v=n+k w$ is the overall right-moving momentum.

Applying the same logic to the expressions for the $N>1$ case, we notice many similarities and a few important differences. One can read off a few features immediately from the remainder term in (3.47): there is a single radial momentum $s$; the measure of the integral is identical to the cigar case and is determined by a linear combination of the radial momentum $s$ and the overall right-moving angular momentum $v$. The asymptotic geometry of the target space sigma model is therefore the combination of a radial direction, an overall asymptotic circle, which is the superpartner of the radial direction, and a compact section. The quantum numbers $n$ and $w$ are interpreted as the momentum and winding along the asymptotic circle direction. In the cigar geometry with asymptotics $\mathbb{R} \times S^{1}$, the left and right moving momenta along the circle are decoupled. The lack of decoupling for the $N>1$ cases shows that the asymptotic circle direction is fibered over the compact section. All these features are in perfect agreement with the asymptotic geometry of the space (4.1). A more detailed analysis should show that even the precise compact section is coded in the degeneracies in the remainder term.

Summary. We have shown that our elliptic genus includes the states that were counted in the twisted index calculation of [11]. Moreover, we argued that the remainder term is 
in accord with the asymptotic geometry of the models. Combined with the modular and elliptic properties of our proposal (which gives the central charge), as well as the heuristics based on the gauged linear sigma model, we believe that this provides good evidence for our identification of the conformal field theory that leads to the Jacobi forms (2.2) as the sigma model on the asymptotic linear dilaton background (4.1).

\section{$5 \quad$ Elliptic genus of generalized Liouville theories}

The elliptic genus of Liouville theory [6] also allows for a generalization to a family labeled by an extra integer $N$. We summarize the salient features of the proposal in this case. Many of the technical details are omitted since the calculations follow closely those that we have presented in sections 2 and 3 . We begin with the following proposal:

$$
\chi_{L_{N}}\left(\tau, \alpha, \beta_{i}\right)=\int_{0}^{1} d s_{1} d s_{2} \sum_{m, w} \prod_{i=1}^{N}\left[\frac{\theta_{11}\left(\tau, s_{1} \tau+s_{2}-\alpha-\frac{N \alpha}{k}+\beta_{i}\right)}{\theta_{11}\left(\tau, s_{1} \tau+s_{2}-\frac{N \alpha}{k}+\beta_{i}\right)}\right] e^{\frac{2 \pi i N \alpha w}{k}} e^{-\frac{\pi}{k \tau 2}\left|m+\tau w+k\left(s_{1} \tau+s_{2}\right)\right|^{2}} .
$$

This is a generalization of the elliptic genus of Liouville theory [6]. We have twisted by $N$ $\mathrm{U}(1)$ global symmetries. In order to derive the modular and elliptic properties, we do a Poisson resummation to obtain

$$
\begin{aligned}
\chi_{L_{N}}\left(\tau, \alpha, \beta_{i}\right)=k \int_{0}^{1} d s_{1} d s_{2} \sum_{m, w} \prod_{i=1}^{N}\left[\frac{\theta_{11}\left(\tau, s_{1} \tau+s_{2}-\alpha-\frac{N \alpha}{k}+\beta_{i}\right)}{\theta_{11}\left(\tau, s_{1} \tau+s_{2}-\frac{N \alpha}{k}+\beta_{i}\right)}\right] \\
\times e^{-2 \pi i k s_{2} w} e^{2 \pi i s_{1}(k m-N \alpha)} e^{-\frac{\pi k}{\tau_{2}}\left|m-\frac{N \alpha}{k}+w \tau\right|^{2}} .
\end{aligned}
$$

The modular and elliptic properties can be checked to be same as those found in equation (2.12), with the same central charge. We therefore have another path integral expression for a real Jacobi form with matrix index.

As was shown in $[9,10]$ the elliptic genus of the cigar and Liouville models (at the same asymptotic radius $\sqrt{k \alpha^{\prime}}$ ) are related by a $\mathbb{Z}_{k}$ orbifold. Analogously, we will show later in the section that the above two models are related by a $\mathbb{Z}_{\frac{k}{N}}$ orbifold. But first we obtain the holomorphic and remainder pieces of the Liouville generalization.

\subsection{The mock modular form and its completion}

To extract the holomorphic piece from the path integral expression, the analysis proceeds as before. We perform a single Poisson resummation on equation (5.2) to obtain

$$
\chi_{L_{N}}\left(\tau, \alpha, \beta_{i}\right)=\sqrt{k \tau_{2}} \int_{0}^{1} d s_{1} d s_{2} \sum_{n, w} \prod_{i=1}^{N}\left[\frac{\theta_{11}\left(s_{1} \tau+s_{2}-\alpha-\frac{N \alpha}{k}+\beta_{i}, \tau\right)}{\theta_{11}\left(s_{1} \tau+s_{2}-\frac{N \alpha}{k}+\beta_{i}, \tau\right)}\right] e^{\frac{2 \pi i N \alpha n}{k}} e^{-2 \pi i k s_{2} w} q^{\ell_{0}} \bar{q}^{\bar{\ell}_{0}},
$$

where $\ell_{0}$ and $\bar{\ell}_{0}$ are given by

$$
\ell_{0}=\frac{\left(n+k\left(s_{1}-w\right)\right)^{2}}{4 k} \quad \bar{\ell}_{0}=\frac{\left(n+k\left(s_{1}+w\right)\right)^{2}}{4 k} .
$$

We expand the theta functions in power series using equations (3.3) and (3.6); the holonomy integral over $s_{2}$ now leads to the constraint

$$
\sum_{i}\left(r_{i}-m_{i}+1\right)=k w .
$$


Substituting the constraint into the expression gives

$$
\begin{aligned}
\chi_{L_{N}}\left(\tau, \alpha, \beta_{i}\right)=(-1)^{N} \frac{\sqrt{k \tau_{2}}}{\eta^{3 N}(\tau)} \sum_{m_{i}, r_{i}, n, w} \int_{0}^{1} d s_{1}(-1)^{\sum_{i} m_{i}} q^{\frac{1}{2} \sum\left(m_{i}-\frac{1}{2}\right)^{2}} z^{\sum_{i}\left(m_{i}-\frac{1}{2}\right)} \\
q^{-n w} z^{\frac{N}{k}(n-k w)}(q \bar{q})^{\bar{\ell}_{0}} \delta_{\sum_{i}\left(r_{i}-m_{i}+1\right)-k w} \prod_{i=1}^{N} y_{i}^{r_{i}-m_{i}+1} S_{r_{i}} .
\end{aligned}
$$

We perform the integral over the holonomy $s_{1}$ by first introducing a radial momentum $s$ (in order to linearize the $s_{1}$ exponent). We also introduce the right-moving momentum variable $v=n+k w$ and follow the steps that were followed for the models in section 3 . We obtain a sum over $2^{N}+1$ terms, out of which precisely two terms combine to produce a contour integral which can be performed to give the purely holomorphic part of the elliptic genus:

$$
\chi_{L_{N}, h o l}\left(\tau, \alpha, \beta_{i}\right)=\left(\frac{i \theta_{11}(\tau,-\alpha)}{\eta(\tau)^{3}}\right)^{N} \sum_{w \in \mathbb{Z}} q^{k w^{2}} z^{-2 N w} \sum_{v=0}^{k-1}\left(z^{-\frac{N}{k}} q^{w}\right)^{v} \sum_{p_{i} \in \mathbb{Z}} \delta_{\sum p_{i}-k w} \prod_{i=1}^{N} \frac{y_{i}^{p_{i}}}{\left(1-z^{-1} q^{p_{i}}\right)} .
$$

\subsubsection{The completion}

The remaining $2^{N}-1$ terms make up the remainder function:

$$
\begin{aligned}
\chi_{L_{N}}^{r e m}\left(\tau, \alpha, \beta_{i}\right)=( & \left.\frac{i \theta_{11}(\tau, \alpha)}{\pi \eta^{3}(\tau)}\right)^{N} \sum_{w \in \mathbb{Z}} \sum_{v=-k+1}^{0} q^{k w^{2}-v w} z^{-N\left(2 w-\frac{v}{k}\right)} \\
& \times \sum_{p_{i} \in \mathbb{Z}} \frac{\left(1-z^{-N} q^{k w}\right)}{\prod_{i=1}^{N} y_{i}^{-p_{i}}\left(1-z^{-1} q^{p_{i}}\right)} \delta_{\sum_{i} p_{i}-k w} \int_{\mathbb{R}+i \epsilon} \frac{d s}{2 i s+v}(q \bar{q})^{\frac{v^{2}}{4 k}+\frac{s^{2}}{k}} .
\end{aligned}
$$

\subsubsection{Integral representation}

An integral expression for the holomorphic part can be obtained by writing the delta function as an integral:

$$
\chi_{L_{N}, h o l}\left(\tau, \alpha, \beta_{i}\right)=\frac{1}{2 \pi i} \oint \frac{d x}{x}\left(\frac{i \theta_{11}(\tau,-\alpha)}{\eta(\tau)^{3}}\right)^{N} \sum_{w \in \mathbb{Z}} q^{k w^{2}}\left(z^{-2 N} x^{-k}\right)^{w} \sum_{v=0}^{k-1}\left(z^{-\frac{N}{k}} q^{w}\right)^{v} \sum_{p_{i} \in \mathbb{Z}} \prod_{i=1}^{N} \frac{\left(x y_{i}\right)^{p_{i}}}{\left(1-z^{-1} q^{p_{i}}\right)}
$$

Making use of the formulas (3.38) and (3.40), this can be written in the compact form

$$
\chi_{L_{N}, h o l}\left(\tau, \alpha, \beta_{i}\right)=\frac{1}{2 \pi i} \oint \frac{d x}{x} \sum_{v=0}^{k-1} q^{-\frac{v^{2}}{4 k}} x^{\frac{v}{2}} \Theta_{k, v}\left(\tau,-\gamma-\frac{2 N \alpha}{k}\right) \prod_{i=1}^{N} \frac{\theta_{11}\left(\tau, \gamma+\beta_{i}-\alpha\right)}{\theta_{11}\left(\tau, \gamma+\beta_{i}\right)} .
$$

\subsubsection{Poincaré polynomial}

The Poincaré polynomial of the model is given by the $q \rightarrow 0$ limit of the Liouville elliptic genus. The $\Theta$-function at level $k$ reduces to a single term in this limit. The Poincaré polynomial is $y_{i}$ independent, and equal to:

$$
\chi_{L_{N}, h o l}(\alpha)=\frac{z^{N / 2}-z^{-N / 2}}{1-z^{-N / k}}
$$

Thus, we have $k$ ground states, with R-charges distributed symmetrically around zero, and quantized in units of $N / k$. They carry no global charge. 


\subsubsection{Character interpretation}

In order to find the character representation of the holomorphic piece, we write the delta function constraint in equation (5.7) as

$$
\left(\sum_{i=1}^{N} p_{i}\right)-k w=\sum_{i=1}^{N}\left(p_{i}-M w\right)
$$

where we have used $k=N M$. Defining $r_{i}=p_{i}-M w$, we find that

$$
\chi_{L_{N}, h o l}\left(\tau, \alpha, \beta_{i}\right)=\left(\frac{i \theta_{11}(\tau,-\alpha)}{\eta(\tau)^{3}}\right)^{N} \sum_{w \in \mathbb{Z}} q^{k w^{2}} z^{-2 N w} \sum_{v=0}^{k-1}\left(z^{-\frac{N}{k}} q^{w}\right)^{v} \sum_{r_{i}} \delta_{\sum r_{i}} \prod_{i=1}^{N} \frac{y_{i}^{r_{i}+M w}}{\left(1-z^{-1} q^{r_{i}+M w}\right)} .
$$

Let us now interpret the result in terms of a constrained sum of products of Ramond sector characters. We begin with Ramond ground states with R-charge $Q_{R}$. We take the tensor product of $N$ of these representations, and spectrally flow the individual factors by $-r_{i}$ units to find:

$$
\chi_{\otimes}\left(Q_{R} ;-r_{i} ; q, z\right)=\prod_{i=1}^{N} q^{\left(\frac{c_{f}}{6}-\frac{1}{2}\right) r_{i}^{2}} z^{-\left(\frac{c_{f}}{3}-1\right) r_{i}} z^{Q_{R}-\frac{1}{2}} q^{\left(-Q_{R}+\frac{1}{2}\right) r_{i}} \frac{1}{1-z^{-1} q^{r_{i}}}\left(\frac{i \theta_{11}(\tau,-\alpha)}{\eta^{3}(\tau)}\right)^{N}
$$

Now assume that $-Q_{R}+1 / 2=v / k-r_{i} / M$ in each of the $N$ sectors. We then see that the quadratic term in the exponent cancels. We moreover must assume that $\sum_{i=1}^{N} r_{i}=0$. We thus find, at this stage:

$$
\chi_{\otimes}\left(Q_{R}, r_{i} ; q, z\right)=\left(\frac{i \theta_{11}(\tau,-\alpha)}{\eta^{3}(\tau)}\right)^{N} z^{-\frac{N v}{k}} \delta_{\sum_{i=1}^{N} r_{i}=0} \prod_{i=1}^{N} \frac{1}{1-z^{-1} q^{r_{i}}}
$$

We next perform spectral flow by $-M w$ units in the direct sum of the factor $\mathcal{N}=2$ superconformal algebras. Summing over all $r_{i}$ subject to the delta function constraint, we obtain expression (5.13), and therefore, the character sum interpretation of the holomorphic contribution.

\subsection{Orbifolds}

We will now show that the two classes of models we have discussed so far are related by a $\mathbb{Z}_{M}$ orbifold, where the discrete orbifold group is a subgroup of the $\mathrm{U}(1) R$-symmetry group. There is a systematic way to construct $R$-symmetry orbifolds, following [4]. Starting with the elliptic genus, we first define the "twisted blocks":

$$
\chi_{m_{a}, m_{b}}\left(\tau, \alpha, \beta_{i}\right)=(-1)^{\frac{c}{3} m_{a} m_{b}} q^{\frac{c m_{a}^{2}}{6}} z^{m_{a} \frac{c}{3}} \chi\left(\tau, \alpha+m_{a} \tau+m_{b}\right) \prod_{i} y_{i}^{-m_{a}} .
$$

The integers $m_{a}, m_{b} \in \mathbb{Z}_{M}$ label the twisted sectors in the orbifold theory. For the case at hand we work with the holomorphic part of the Liouville elliptic genus, the discussion with 
the remainder can be done in an analogous manner. Using the definition above we obtain the twisted blocks (after a simplification where we shift the $p_{i}$ variable by $m_{a}$ ):

$$
\begin{gathered}
\chi_{L_{N}, m_{a}, m_{b}}\left(\tau, \alpha, \beta_{i}\right)=(-1)^{N\left(m_{a}+m_{b}+m_{a} m_{b}\right)} e^{\frac{2 \pi i m_{b}}{M}\left(N m_{a}-v\right)} q^{\frac{m_{a}^{2} N}{M}} z^{\frac{2 m_{a} N}{M}} \\
\left(\frac{i \theta_{11}(\tau,-\alpha)}{\eta^{3}(\tau)}\right)^{N} \sum_{w \in \mathbb{Z}} \sum_{v=0}^{k-1} q^{k w^{2}} z^{-2 N w}\left(z^{-\frac{N}{k}} q^{w}\right)^{v} q^{-2 w N m_{a}-\frac{v m_{a}}{M}} \\
\sum_{p_{i} \in \mathbb{Z}} \prod_{i=1}^{N} \frac{y_{i}^{p_{i}}}{1-z^{-1} q^{p_{i}}} \delta_{\sum p_{i}-k w+N m_{a}} .
\end{gathered}
$$

It can then be shown, on general grounds (see [4]), that the following sum over the twisted blocks satisfies all the requirements to be the elliptic genus of a conformal field theory with the same central charge:

$$
\chi_{L_{N} ; \mathbb{Z}_{M}}\left(\tau, \alpha, \beta_{i}\right)=\sum_{m_{a}, m_{b} \in \mathbb{Z}_{\mathbb{M}}}(-1)^{N\left(m_{a}+m_{b}+m_{a} m_{b}\right)} \chi_{L_{N}, m_{a}, m_{b}}\left(\tau, \alpha, \beta_{i}\right) .
$$

The sum over the $m_{b}$ variable leads to the constraint $N m_{a}=v$ modulo $M$. The solutions to this can be obtained as follows: write $v=V+j M$, where $V=0,1,2 \ldots M-1$ and $j=0,1,2, \ldots N-1$. We have split the $v$-summation into $N$ intervals, each of length $M$. Then, one can check that for every value of $m_{a}$, there are exactly $N$ solutions to the constraint, one in each of the $N$ sub-intervals of the original $v$-summation. Substituting this into the sum over the twisted blocks, we obtain the result

$$
\begin{aligned}
& \chi_{L_{N}, h o l ; \mathbb{Z}_{M}}\left(\tau, \alpha, \beta_{i}\right)=\left(\frac{i \theta_{11}(\tau,-\alpha)}{\eta^{3}(\tau)}\right)^{N} \sum_{w \in \mathbb{Z}} \sum_{V=0}^{M-1} q^{k w^{2}-v w} z^{-N\left(2 w-\frac{V}{k}\right)} \sum_{j=0}^{N-1} q^{-j M w} z^{j} \\
& \times \sum_{p_{i} \in \mathbb{Z}} \prod_{i=1}^{N} \frac{y_{i}^{p_{i}}}{1-z^{-1} q^{p_{i}}} \delta_{\sum p_{i}-k w+V+j M} \\
& \equiv \chi_{N, h o l}\left(\tau, \alpha, \beta_{i}\right) .
\end{aligned}
$$

In the second equality we have made the identification of the orbifold elliptic genus with the holomorphic part written out in the form of equation (3.27). A similar calculation can be performed to show that the remainder functions are also related by a $\mathbb{Z}_{M}$ orbifold. We therefore find that, much like the original case of the Liouville and cigar theories at the same asymptotic radius, the two path integrals in (2.2) and (5.1) are related by an overall $\mathbb{Z}_{M}$ orbifold.

\section{Conclusions}

In this paper we have exhibited interesting real Jacobi forms with matrix index parameterized by two integers $k$ and $N$. For a given value of these parameters we have provided evidence that the Jacobi form arises as the elliptic genus of a non-compact conformal field theory with central charge $c=3 N(1+2 N / k)$. There is a geometric description of 
this conformal field theory as a complex $N$-dimensional Kähler manifold that has an $S^{1}$ fibered over the complex projective space $\mathbb{C P}^{N-1}$, along with a radial direction which has an asymptotic linear dilaton. When $N$ divides $k$, we also showed that a $\mathbb{Z}_{\frac{k}{N}}$ orbifold of our proposal gave rise to another class of elliptic genera with the same central charge. These can be understood as multi-variable generalizations of Liouville theories that also have a gauged linear sigma model description [13]. Indeed, we expect that our techniques apply to the whole zoo of models described in $[13,14]$. Moreover, the identification of the elliptic genera of this large class of models opens a window onto their full spectrum.

The conformal field theory backgrounds that we studied appear in string theory when we consider the worldsheet description of $N S 5$ branes wrapped on $\mathbb{C P}^{N-1}$. Thanks to our results, it has become straightforward to calculate the worldsheet elliptic genera in such backgrounds. An application of these results is to compute a space-time index that arises from the worldsheet (generalized) elliptic genus through integration over the fundamental domain. This potentially generates interesting mock modular forms with a direct spacetime interpretation. In [32] an example of this technique was exhibited, by considering the near horizon geometry of two NS5 branes wrapping a $K 3$ surface. The worldsheet theory which describes such a background includes the cigar elliptic genus; it will be interesting to generalize these results to other models at $N=1$ as well as to higher $N$ - we have only seen the beginning of these applications.

A first principles derivation of the elliptic genus is desirable. It should be attainable by applying localization techniques to the gauged linear sigma model (GLSM) description of these backgrounds $[13,14]$. This is especially interesting because there are other non-compact backgrounds that also have a gauged linear sigma model description, such as the Euclidean Taub-NUT background to which our techniques could be adapted. A further challenge includes the understanding of a conjectured elliptic genus of Atiyah-Hitchin space [33].

Our elliptic genera exhibit shadows which (like those of the orbifolded tensor product models of [18]) suggest that the realm of mock modular forms may be usefully defined even beyond the class that is at present mathematically well-understood [30]. Producing qualitatively new examples of non-compact elliptic genera, as we did, should be helpful in coming to grips with a grand synthesis.

\section{Acknowledgments}

We would like to thank Atish Dabholkar, Nima Doroud, Jaume Gomis, Dan Israel, Bruno Le Floch, Sameer Murthy, Suresh Nampuri and Giuseppe Policastro for useful and interesting discussions. This work was supported in part by the ANR grant ANR-09-BLAN0157-02. 


\section{A Formulas}

\section{A.1 Definitions and properties}

The Jacobi theta function is given by

$$
\theta_{11}(\tau, \alpha)=-i \sum_{n=-\infty}^{\infty}(-1)^{n} q^{\frac{1}{2}\left(n-\frac{1}{2}\right)^{2}} z^{n-\frac{1}{2}}
$$

where $q=e^{2 \pi i \tau}$ and $z=e^{2 \pi i \alpha}$. The Dedekind eta function is given by

$$
\eta(\tau)=q^{\frac{1}{24}} \prod_{n=1}^{\infty}\left(1-q^{n}\right) .
$$

The modular and elliptic properties of the combination $\theta_{11}(\tau, \alpha) / \eta^{3}(\tau)$ are:

$$
\begin{aligned}
& \frac{\theta_{11}}{\eta^{3}}\left(\frac{a \tau+b}{c \tau+d}, \frac{\alpha}{c \tau+d}\right)=(c \tau+d)^{-1} e^{\pi i \frac{c \alpha^{2}}{c \tau+d}} \frac{\theta_{11}}{\eta^{3}}(\tau, \alpha) . \\
& \frac{\theta_{11}}{\eta^{3}}\left(\tau, \alpha+m_{a} \tau+m_{b}\right)=(-1)^{m_{a}+m_{b}} q^{-\frac{m_{a}^{2}}{2}} z^{-m_{a}} \frac{\theta_{11}}{\eta^{3}}(\tau, \alpha) .
\end{aligned}
$$

We also use the level $k$ theta function, defined to be

$$
\Theta_{k, v}(\tau, \alpha)=\sum_{j \in \mathbb{Z}+\frac{v}{2 k}} q^{k j^{2}} z^{k j}
$$

\section{A.2 Identities and their proof}

In this subsection of the appendix, we list a number of identities, and their proofs. We use the series

$$
S_{r}(q)=\sum_{n=0}^{\infty}(-1)^{n} q^{\frac{n(n+2 r+1)}{2}},
$$

which is related to the inverse of the $\theta_{11}$ function by:

$$
\frac{1}{i \theta_{11}(\tau, \alpha)}=\frac{1}{\eta^{3}(\tau)} \sum_{r \in \mathbb{Z}} z^{r+\frac{1}{2}} S_{r}(q)
$$

when the arguments satisfy $|q|<|z|<1$. This identity can be proven by matching coefficients of powers of $z$ on both sides of the identity. Indeed, let's consider the contour integral:

$$
C(q)=\frac{1}{2 \pi i} \oint \frac{d z}{z^{r+\frac{3}{2}}} \frac{1}{i \theta_{11}(\tau, \alpha)}
$$

where the contour lies in the annulus $|q|<|z|<1$ and circles the origin counter clockwise once. We use the product form of the Jacobi theta function to find:

$$
C(q)=-q^{-\frac{1}{8}} \frac{1}{2 \pi i} \oint \frac{d z}{z^{r+\frac{3}{2}}} \frac{1}{\left(z^{\frac{1}{2}}-z^{-\frac{1}{2}}\right) \prod_{n=1}^{\infty}\left(1-q^{n}\right)\left(1-z q^{n}\right)\left(1-z^{-1} q^{n}\right)} .
$$


We can compute the integral by picking up the poles inside the contour. These lie at $z=q^{m}$ where $m \in\{1,2,3, \ldots\}$. We pick up the residues at these poles, which are equal to:

$$
R_{m}(q)=\frac{(-1)^{m-1} q^{-m r+m(m-1) / 2}}{\eta^{3}(\tau)} .
$$

We then find the result of the contour integration:

$$
\begin{aligned}
C(q) & =\sum_{m=1,2, \ldots}^{\infty} \frac{(-1)^{m-1} q^{-m r+m(m-1) / 2}}{\eta^{3}(\tau)} \\
& =\sum_{m=0,1, \ldots}^{\infty} \frac{(-1)^{m} q^{-m r+m(m+1) / 2} q^{-r}}{\eta^{3}(\tau)} \\
& =\frac{1}{\eta^{3}(\tau)} q^{-r} S_{-r}(q)=\frac{1}{\eta^{3}(\tau)} S_{r}(q) .
\end{aligned}
$$

In the last line, we use the equality:

$$
q^{-r} S_{-r}(q)=S_{r}(q)
$$

which can be proven by observing that the difference of these two expressions is a finite sum whose terms cancel two by two. We thus have proven the desired equality, in the particular range of arguments. Let us also observe that the equality:

$$
S_{r}(q)+S_{-r-1}(q)=1
$$

is now easily proven by shifting the summation variable in $S_{-r-1}$ by one and using equation (A.12). Alternatively, these identities satisfied by the series $S_{r}$ can be found by using properties of the $\theta_{11}$-function, and contour integration.

Another identity we put to good use is the expansion:

$$
\frac{i \theta_{11}(\tau, \alpha)}{1-z q^{p}}=\sum_{m \in \mathbb{Z}}(-1)^{m} q^{\frac{1}{2}\left(m-\frac{1}{2}\right)^{2}} z^{m-\frac{1}{2}} S_{-m+p}(q) .
$$

We can prove this identity, for instance for $\left|z q^{p}\right|<1$, by expanding the denominator and then rearranging terms, as follows:

$$
\begin{aligned}
\frac{i \theta_{11}(\tau, \alpha)}{1-z q^{p}} & =\sum_{m \in \mathbb{Z}}(-1)^{m} q^{\frac{\left(m-\frac{1}{2}\right)^{2}}{2}} z^{m-\frac{1}{2}} \sum_{l=0,1, \ldots}^{\infty}\left(z q^{p}\right)^{l} \\
& =\sum_{m=-\infty}^{+\infty}(-1)^{m} q^{\frac{\left(m-\frac{1}{2}\right)^{2}}{2}} z^{m-\frac{1}{2}} \sum_{l=0,1, \ldots}^{\infty} q^{(p-m) l+l(l+1) / 2} \\
& =\sum_{m=-\infty}^{+\infty}(-1)^{m} q^{\frac{\left(m-\frac{1}{2}\right)^{2}}{2}} z^{m-\frac{1}{2}} S_{-m+p}(q) .
\end{aligned}
$$

In going from the first to the second line, we have shifted the summation variable $m$ by $-l$. For $\left|z q^{p}\right|>1$, the identity can be proven analogously. 
Open Access. This article is distributed under the terms of the Creative Commons Attribution License (CC-BY 4.0), which permits any use, distribution and reproduction in any medium, provided the original author(s) and source are credited.

\section{References}

[1] A. Schellekens and N. Warner, Anomalies and modular invariance in string theory, Phys. Lett. B 177 (1986) 317 [INSPIRE].

[2] E. Witten, Elliptic genera and quantum field theory, Commun. Math. Phys. 109 (1987) 525 [INSPIRE].

[3] T. Eguchi, H. Ooguri, A. Taormina and S.-K. Yang, Superconformal algebras and string compactification on manifolds with $\mathrm{SU}(N)$ holonomy, Nucl. Phys. B 315 (1989) 193 [INSPIRE].

[4] T. Kawai, Y. Yamada and S.-K. Yang, Elliptic genera and $N=2$ superconformal field theory, Nucl. Phys. B 414 (1994) 191 [hep-th/9306096] [INSPIRE].

[5] E. Witten, On the Landau-Ginzburg description of $N=2$ minimal models, Int. J. Mod. Phys. A 9 (1994) 4783 [hep-th/9304026] [INSPIRE].

[6] J. Troost, The non-compact elliptic genus: mock or modular, JHEP 06 (2010) 104 [arXiv: 1004.3649] [INSPIRE].

[7] S. Zwegers, Mock theta functions, Ph.D. thesis, Utrecht University, Utrecht The Netherlands (2002).

[8] D. Zagier, Ramanujan's mock theta functions and their applications d'après Zwegers and Bringmann-Ono, Séminaire Bourbaki 986, Astérisque France (2007).

[9] T. Eguchi and Y. Sugawara, Non-holomorphic modular forms and $\mathrm{SL}(2, R) / \mathrm{U}(1)$ superconformal field theory, JHEP 03 (2011) 107 [arXiv: 1012.5721] [INSPIRE].

[10] S.K. Ashok and J. Troost, A twisted non-compact elliptic genus, JHEP 03 (2011) 067 [arXiv: 1101.1059] [inSPIRE].

[11] S.K. Ashok, S. Nampuri and J. Troost, Counting strings, wound and bound, JHEP 04 (2013) 096 [arXiv: 1302.1045] [INSPIRE].

[12] E. Kiritsis, C. Kounnas and D. Lüst, A large class of new gravitational and axionic backgrounds for four-dimensional superstrings, Int. J. Mod. Phys. A 9 (1994) 1361 [hep-th/9308124] [INSPIRE].

[13] K. Hori and A. Kapustin, Duality of the fermionic $2 D$ black hole and $N=2$ Liouville theory as mirror symmetry, JHEP 08 (2001) 045 [hep-th/0104202] [INSPIRE].

[14] K. Hori and A. Kapustin, World sheet descriptions of wrapped NS five-branes, JHEP 11 (2002) 038 [hep-th/0203147] [INSPIRE].

[15] C. Ziegler, Jacobi forms of higher degree, Abh. Math. Semi. Univ. Hamburg 59 (1989) 191.

[16] N.-P. Skoruppa, Jacobi forms of critical weight and Weil representations, arXiv:0707.0718.

[17] A. Semikhatov, A. Taormina and I.Y. Tipunin, Higher level Appell functions, modular transformations and characters, math.QA/0311314 [INSPIRE].

[18] S.K. Ashok and J. Troost, Elliptic genera of non-compact Gepner models and mirror symmetry, JHEP 07 (2012) 005 [arXiv: 1204.3802] [INSPIRE]. 
[19] K. Miki, The representation theory of the $\mathrm{SO}(3)$ invariant superconformal algebra, Int. J. Mod. Phys. A 5 (1990) 1293 [inSPIRE].

[20] T. Eguchi and Y. Sugawara, Modular bootstrap for boundary $N=2$ Liouville theory, JHEP 01 (2004) 025 [hep-th/0311141] [INSPIRE].

[21] D. Israel, A. Pakman and J. Troost, Extended $\mathrm{SL}(2, R) / \mathrm{U}(1)$ characters, or modular properties of a simple nonrational conformal field theory, JHEP 04 (2004) 043 [hep-th/0402085] [INSPIRE].

[22] S. Elitzur, A. Forge and E. Rabinovici, Some global aspects of string compactifications, Nucl. Phys. B 359 (1991) 581 [INSPIRE].

[23] G. Mandal, A.M. Sengupta and S.R. Wadia, Classical solutions of two-dimensional string theory, Mod. Phys. Lett. A 6 (1991) 1685 [inSPIRE].

[24] E. Witten, On string theory and black holes, Phys. Rev. D 44 (1991) 314 [INSPIRE].

[25] F. Benini, R. Eager, K. Hori and Y. Tachikawa, Elliptic genera of $2 d N=2$ gauge theories, arXiv: 1308.4896 [INSPIRE].

[26] A. Gadde and S. Gukov, $2 d$ index and surface operators, arXiv:1305.0266 [INSPIRE].

[27] B. Haghighat, A. Iqbal, C. Kozcaz, G. Lockhart and C. Vafa, M-strings, arXiv:1305.6322 [INSPIRE].

[28] V.G. Kac and M. Wakimoto, Integrable highest weight modules over affine superalgebras and number theory, hep-th/9407057 [INSPIRE].

[29] M. Stern and P. Yi, Counting Yang-Mills dyons with index theorems, Phys. Rev. D 62 (2000) 125006 [hep-th/0005275] [INSPIRE].

[30] A. Dabholkar, S. Murthy and D. Zagier, Quantum black holes, wall crossing and mock modular forms, arXiv:1208.4074 [INSPIRE].

[31] T. Eguchi, H. Ooguri and Y. Tachikawa, Notes on the K3 surface and the Mathieu group $M_{24}$, Exper. Math. 20 (2011) 91 [arXiv:1004.0956] [INSPIRE].

[32] J.A. Harvey and S. Murthy, Moonshine in fivebrane spacetimes, arXiv:1307.7717 [INSPIRE].

[33] B. Haghighat, J. Manschot and S. Vandoren, A $5 d / 2 d / 4 d$ correspondence, JHEP 03 (2013) 157 [arXiv:1211.0513] [INSPIRE]. 